\title{
Application of Fuzzy Finite Difference Scheme for the Non-homogeneous Fuzzy Heat Equation
}

\section{Samaneh Zabihi}

Islamic Azad University Central Tehran Branch

reza ezzati ( $\nabla$ ezati@kiau.ac.ir )

Islamic Azad University-Karaj Branch https://orcid.org/0000-0002-3162-6212

\section{F Fattahzadeh}

Islamic Azad University Central Tehran Branch

\section{J Rashidinia}

Iran University of Science and Technology

\section{Research Article}

Keywords: Forward fuzzy difference scheme, Backward fuzzy difference scheme, Centered fuzzy finite difference scheme, Generalized Hukuhara difference, Non-homogeneous initial-boundary fuzzy heat equation

Posted Date: October 27th, 2021

DOI: https://doi.org/10.21203/rs.3.rs-1003065/v1

License: (c) (i) This work is licensed under a Creative Commons Attribution 4.0 International License. Read Full License

Version of Record: A version of this preprint was published at Soft Computing on January 30th, 2022. See the published version at https://doi.org/10.1007/s00500-021-06670-9. 
Soft Computing manuscript No.

(will be inserted by the editor)

\title{
Application of fuzzy finite difference scheme for the non-homogeneous fuzzy heat equation
}

\author{
S. Zabihi · R. Ezzati * F. Fattahzadeh • J. \\ Rashidinia
}

Received: date / Accepted: date

\begin{abstract}
A numerical framework based on fuzzy finite difference is presented for approximating fuzzy triangular solutions of fuzzy partial differential equations by considering the type of $[g H-p]$-differentiability. The fuzzy triangle functions are expanded using full fuzzy Taylor expansion to develop a new fuzzy finite difference method. By considering the type of gH-differentiability, we approximate the fuzzy derivatives with a new fuzzy finite-difference. In particular, we propose using this method to solve non-homogeneous fuzzy heat equation with triangular initial-boundary conditions. We examine the truncation error and the convergence conditions of the proposed method. Several numerical examples are presented to demonstrate the performance of the methods. The final results demonstrate the efficiency and the ability of the new fuzzy finite difference method to produce triangular fuzzy numerical results which are more consistent with existing reality.
\end{abstract}

Keywords Forward fuzzy difference scheme, Backward fuzzy difference scheme, Centered fuzzy finite difference scheme, Generalized Hukuhara difference, Non-homogeneous initial-boundary fuzzy heat equation.

Mathematics Subject Classification (2010) 03E72 $\cdot 46$ S40 $\cdot 32$ W50 $\cdot 35$ K05

S. Zabihi

Department of Mathematics, Central Tehran Branch, Islamic Azad University, Tehran, Iran.

R. Ezzati (Corresponding Author)

Department of Mathematics, Karaj Branch, Islamic Azad University, Karaj, Iran.

E-mail: ezati@kiau.ac.ir

F. Fattahzadeh

Department of Mathematics, Central Tehran Branch, Islamic Azad University, Tehran, Iran.

J. Rashidinia

Department of Mathematics, Central Tehran Branch, Islamic Azad University, Tehran, Iran. 


\section{Introduction}

In recent decades, fuzzy set theory has been proven to be a useful tool for modeling systems with uncertainties, giving the models a more realistic look at reality and enabling them to express themselves with a more comprehensive outlook.

The fuzzy derivative concept first appeared in Chang and Zadeh's [14]. Hukuhara's paper [16] is the starting point for the set-valued and fuzzy differential equations. Puri and Ralescu [23] suggested the fuzzy differential equations modeling with uncertainty under the concept of H-differentiability. Further studies developed fuzzy differential equations based on the Hukuhara derivative, such as those presented by Kaleva in [18]. There are some fuzzy differential equations in this framework, however, for which the diameter of the solution increases as the time $t$ increases [15].

To overcome this shortcoming, Bede and Gal introduced the weakly generalized differentiability and the strongly generalized differentiability for the fuzzy functions [7]. Moreover, they presented a more general definition of derivatives for the fuzzy functions and their applications for solving fuzzy differential equations $[7,8]$. Stefanini and Bede introduced generalized Hukuhara differentiability (gH-differentiability) [25] for interval-valued functions by using the concept of generalization of the Hukuhara difference of compact convex set. They showed that this concept of differentiability has relationships with weakly generalized differentiability and strongly generalized differentiability. The disadvantage of the strongly generalized differentiability of a function compared to H-differentiability is that in this case the fuzzy differential equation has no unique solution [7]. Also, in [12] the authors studied relationships between the strongly generalized differentiability and the gH-differentiability , showing the equivalence between these two concepts when the set of switching points of the interval-valued function is finite. Recently, Y.Chalco-Cano et al. [13] provided a new characterization of the switching points for $\mathrm{gH}$ differentiability and shown that the set of all switching points is at most countable.

Partial differential equations explain the majority of phenomena in the fields of mathematics, physics, and engineering. However, mathematical modeling of these phenomena requires a wide variety of data and information. Unfortunately, the measurement of these variables is inherently uncertain. Therefore, the fuzzy partial differential equation is a useful tool for modeling systems with uncertainties [11,1,2,10,22,5,17].

For many fuzzy partial differential equations, analytical solutions are challenging to obtain. Consequently, it is crucial to create some reliable and efficient methods for solving fuzzy partial differential equations. Numerous researchers are presently focusing on the numerical solution of fuzzy partial differential equations, such as difference method [1,4], Adomian method [2, 24], finite volume method [21], etc.

In recent years, there has been an increase in interest in the use of the finite difference method to solve fuzzy partial differential equations. According 
to our knowledge, all papers that have used this method have rewritten the fuzzy partial differential equation as two crisp partial differential equations and solved them using the usual finite difference method. In comparison, this paper is devoted to developing a new fuzzy finite difference method through fuzzy arithmetic and fuzzy Taylor expansion. We approximate the fuzzy derivatives with a fuzzy finite-difference by considering the type of gH-differentiability. The fuzzy numerical solution of the fuzzy partial differential equations can be obtained without implicitly embedding them into crisp equations through our method. Even though this paper deals with the fuzzy heat equation, our method can be used to find the numerical solution for a wide variety of fuzzy partial differential equations.

Now, let's take a quick look at the contents. Section 2 presents some concepts related to fuzzy numbers and generalized Hukuhara differentiability, as well as some theorems and lemmas used in the central part of the paper. The fuzzy finite difference method for one variable fuzzy functions is discussed in Section 3 and we obtain different formulas for forward, backward, and central difference depending on the type of $g H$-differentiability. Taking into account the type of $[g H-p]$-differentiability, we show corresponding formulas for the fuzzy finite difference method of the non-homogeneous heat equation in Section 4. Further, we describe and analyze in detail the convergence condition of the method as well as truncation error. A full description is given for one of the three examples in Section 5. The last section of the paper discusses conclusions, applications, and future possibilities.

\section{Preliminaries}

The purpose of this section is to introduce the general terms and definitions used to describe fuzzy operations and the necessary notations.

The triangular fuzzy number $a \in \mathbb{R}_{\mathscr{T}}$ is defined as an ordered triple $a=$ $\left(a_{1}, a_{2}, a_{3}\right)$ with $a_{1} \leq a_{2} \leq a_{3}$. Some properties of the triangular fuzzy number are discussed in [19], but we will describe some of the properties of this class of numbers here which are used in this paper.

Definition 21 (See [9], [6]) The generalized Hukuhara difference of two fuzzy numbers $a, b \in \mathbb{R}_{F}$ is the fuzzy number $c$, (if it exists), such that

$$
a \ominus_{g H} b=c \Longleftrightarrow\left\{\begin{array}{c}
(i) \cdot a=b \oplus c ; \\
\text { or }(i i) . b=a \oplus(-1) c .
\end{array}\right.
$$

Now consider $a, b \in \mathbb{R}_{\mathscr{T}}$, then

$$
a \ominus_{g H} b=c \Longleftrightarrow\left\{\begin{array}{r}
(i) \cdot c=\left(a_{1}-b_{1}, a_{2}-b_{2}, a_{3}-b_{3}\right) ; \\
\text { or }(i i) . c=\left(a_{3}-b_{3}, a_{2}-b_{2}, a_{1}-b_{1}\right) .
\end{array}\right.
$$

provided that $c$ is a triangular fuzzy number. 
Remark 22 In the rest of this paper, all fuzzy numbers and fuzzy functions will be considered triangular. Additionally, all the lemmas and theorems will be proved on the assumption that the generalized Hukuhara difference exists.

Proposition 23 Consider $a, b$ and $c$ are triangular fuzzy numbers and Hukuhara difference exists, then

1. $a \ominus(-1) b=\left(a_{1}+b_{3}, a_{2}+b_{2}, a_{3}+b_{1}\right)$ provided that $a \ominus(-1) b$ is a triangular fuzzy number.

2. If $a=c \ominus(-1) b$ then $b=-1(c \ominus a)$.

Proof Case 1. We have

$$
\begin{aligned}
a \ominus(-1) b & =\left(a_{1}, a_{2}, a_{3}\right) \ominus\left(-b_{3},-b_{2},-b_{1}\right) \\
& =\left(a_{1}+b_{3}, a_{2}+b_{2}, a_{3}+b_{1}\right) .
\end{aligned}
$$

Case 2. According to assumption $a=c \ominus(-1) b$ and Hukuhara difference exists, so

$$
\begin{aligned}
a & =c \ominus(-1) b \\
\left(a_{1}, a_{2}, a_{3}\right) & =\left(c_{1}, c_{2}, c_{3}\right) \ominus(-1)\left(b_{1}, b_{2}, b_{3}\right) \\
& =\left(c_{1}, c_{2}, c_{3}\right) \ominus\left(-b_{3},-b_{2},-b_{1}\right)=\left(c_{1}+b_{3}, c_{2}+b_{2}, c_{3}+b_{1}\right) .
\end{aligned}
$$

So

$$
\begin{aligned}
& a_{1}=c_{1}+b_{3}, \Longrightarrow a_{1}-c_{1}=b_{3}, \\
& a_{2}=c_{2}+b_{2}, \Longrightarrow a_{2}-c_{2}=b_{2}, \\
& a_{3}=c_{3}+b_{1}, \Longrightarrow a_{3}-c_{3}=b_{1} .
\end{aligned}
$$

Then

$$
\begin{aligned}
b & =\left(b_{1}, b_{2}, b_{3}\right)=\left(a_{3}-c_{3}, a_{2}-c_{2}, a_{1}-c_{1}\right) \\
& =(-1)\left(c_{1}-a_{1}, c_{2}-a_{2}, c_{3}-a_{3}\right) \\
& =(-1)(c \ominus a) .
\end{aligned}
$$

Thus, the proof is complete.

Proposition 24 Let $\lambda_{1}$ and $\lambda_{2}$ are two real constants such that $\lambda_{1}, \lambda_{2} \geq 0$ (or $\left.\lambda_{1}, \lambda_{2} \leq 0\right)$. If $y(t)$ is a triangular fuzzy function, then

$$
\lambda_{1} y(t) \ominus_{g H} \lambda_{2} y(t)=\left(\lambda_{1}-\lambda_{2}\right) y(t)
$$

Proof First consider $\lambda_{1}$ and $\lambda_{2}$ are positive constants, then

$$
\lambda_{1} y(t)=\left(\lambda_{1} y_{1}(t), \lambda_{1} y_{2}(t), \lambda_{1} y_{3}(t)\right), \quad \lambda_{2} y(t)=\left(\lambda_{2} y_{1}(t), \lambda_{2} y_{2}(t), \lambda_{2} y_{3}(t)\right) .
$$

Now, we have two cases 
i. If $\lambda_{1} \geq \lambda_{2}$, we have

$$
\begin{aligned}
\lambda_{1} y(t) \ominus_{g H} \lambda_{2} y(t) & =\left(\left(\lambda_{1}-\lambda_{2}\right) y_{1}(t),\left(\lambda_{1}-\lambda_{2}\right) y_{2}(t),\left(\lambda_{1}-\lambda_{2}\right) y_{3}(t)\right) \\
& =\left(\lambda_{1}-\lambda_{2}\right) y(t) .
\end{aligned}
$$

ii. If $\lambda_{1} \leq \lambda_{2}$, therefore

$$
\begin{aligned}
\lambda_{1} y(t) \ominus_{g H} \lambda_{2} y(t) & =\left(\left(\lambda_{1}-\lambda_{2}\right) y_{3}(t),\left(\lambda_{1}-\lambda_{2}\right) y_{2}(t),\left(\lambda_{1}-\lambda_{2}\right) y_{1}(t)\right) \\
& =\left(\lambda_{1}-\lambda_{2}\right)\left(y_{1}(t), y_{2}(t), y_{3}(t)\right) \\
& =\left(\lambda_{1}-\lambda_{2}\right) y(t) .
\end{aligned}
$$

Hence, we have Eq.(1). The other case ( $\lambda_{1}$ and $\lambda_{2}$ are negative constants) can be proved in a similar way and we omit the details.

Definition 25 (See [9]) Let $y:(a, b) \rightarrow \mathbb{R}_{\mathscr{T}}$ is a fuzzy-valued function such that $y(t)=\left(y_{1}(t), y_{2}(t), y_{3}(t)\right)$, where $y_{1}(t), y_{2}(t)$ and $y_{3}(t)$ are real-valued differentiable functions on $(a, b)$. Then $y$ is a $[(i)-g H]$-differentiable function at $t_{0} \in(a, b)$ if and only if

$$
y_{g H}^{\prime}\left(t_{0}\right)=\left(y_{1}^{\prime}(t), y_{2}^{\prime}(t), y_{3}^{\prime}(t)\right),
$$

defines a triangular fuzzy number. Similarly, $y$ is a $[(i i)-g H]$-differentiable function at $t_{0}$ if and only if

$$
y_{g H}^{\prime}\left(t_{0}\right)=\left(y_{3}^{\prime}(t), y_{2}^{\prime}(t), y_{1}^{\prime}(t)\right),
$$

is a triangular fuzzy number. In general, if $y(t)$ is a $[(i)-g H]-$ or $[(i i)-g H]-$ differentiable for all $t_{0} \in(a, b)$, then $y$ is generalized Hukuhara differentiable function on $(a, b)$.

Remark 26 We assume that the notations $\mathscr{C}_{g H}^{k}\left([a, b], \mathbb{R}_{\mathscr{T}}\right)$ is stand for all triangular fuzzy function $f$ and it's first $k$, gH-derivatives which are defined on $[a, b]$ and fuzzy continuous [3]. Throughout the rest of this paper, $y(t) \in$ $\mathscr{C}_{g H}^{j}\left([a, b], \mathbb{R}_{\mathscr{T}}\right)$ for $j=1, \ldots, n-1$ and $t \in[a, b]$ with no switching point on $[a, b]$. Moreover, for simplicity

- When $y_{g H}^{(j)}(t)=\left(y_{1}^{(j)}(t), y_{2}^{(j)}(t), y_{3}^{(j)}(t)\right)$, we will use the notation denote $y_{i . g H}^{(j)}(t)$ to show $y_{g H}^{(j)}(t)$

- When $y_{g H}^{(j)}(t)=\left(y_{3}^{(j)}(t), y_{2}^{(j)}(t), y_{1}^{(j)}(t)\right)$, we will use the notation denote $y_{i i . g H}^{(j)}(t)$ to show $y_{g H}^{(j)}(t)$.

(Notice the position of functions $y_{1}^{(j)}(t)$ and $y_{3}^{(j)}(t)$ in these triangular fuzzy functions.) In particular, we have the following cases to show the all kind of gH-differentiability for $y_{g H}^{(j)}(t)$ of order $j$, when $j=0,1,2$. 
Case 1. If $y(t), y_{g H}^{\prime}(t)$ and $y_{g H}^{\prime \prime}(t)$ are $[i-g H]$-differentiable, we have

$$
y_{i . g H}^{\prime}(t)=\left(y_{1}^{\prime}(t), y_{2}^{\prime}(t), y_{3}^{\prime}(t)\right), y_{i . g H}^{\prime \prime}(t)=\left(y_{1}^{\prime \prime}(t), y_{2}^{\prime \prime}(t), y_{3}^{\prime \prime}(t)\right), y_{i . g H}^{\prime \prime \prime}(t)=\left(y_{1}^{\prime \prime \prime}(t), y_{2}^{\prime \prime \prime}(t), y_{3}^{\prime \prime \prime}(t)\right)
$$

Case 2. If $y(t)$ and $y_{g H}^{\prime}(t)$ are $[i-g H]$-differentiable and $y_{g H}^{\prime \prime}(t)$ is $[i i-g H]$-differentiable,

$$
y_{i . g H}^{\prime}(t)=\left(y_{1}^{\prime}(t), y_{2}^{\prime}(t), y_{3}^{\prime}(t)\right), y_{i . g H}^{\prime \prime}(t)=\left(y_{1}^{\prime \prime}(t), y_{2}^{\prime \prime}(t), y_{3}^{\prime \prime}(t)\right), y_{i i . g H}^{\prime \prime \prime}(t)=\left(y_{3}^{\prime \prime \prime}(t), y_{2}^{\prime \prime \prime}(t), y_{1}^{\prime \prime \prime}(t)\right)
$$

Case 3. If $y(t)$ and $y_{g H}^{\prime \prime}(t)$ are $[i-g H]$-differentiable and $y_{g H}^{\prime}(t)$ is $[i i-g H]$-differentiable,

$$
y_{i . g H}^{\prime}(t)=\left(y_{1}^{\prime}(t), y_{2}^{\prime}(t), y_{3}^{\prime}(t)\right), y_{i i . g H}^{\prime \prime}(t)=\left(y_{3}^{\prime \prime}(t), y_{2}^{\prime \prime}(t), y_{1}^{\prime \prime}(t)\right), y_{i \prime . g H}^{\prime \prime \prime}(t)=\left(y_{3}^{\prime \prime \prime}(t), y_{2}^{\prime \prime \prime}(t), y_{1}^{\prime \prime \prime}(t)\right)
$$

Case 4. If $y(t)$ is $[i-g H]$-differentiable and $y_{g H}^{\prime}(t)$ and $y_{g H}^{\prime \prime}(t)$ are $[i i-g H]$-differentiable,

$$
y_{i . g H}^{\prime}(t)=\left(y_{1}^{\prime}(t), y_{2}^{\prime}(t), y_{3}^{\prime}(t)\right), y_{i i . g H}^{\prime \prime}(t)=\left(y_{3}^{\prime \prime}(t), y_{2}^{\prime \prime}(t), y_{1}^{\prime \prime}(t)\right), y_{i . g H}^{\prime \prime \prime}(t)=\left(y_{1}^{\prime \prime \prime}(t), y_{2}^{\prime \prime \prime}(t), y_{3}^{\prime \prime \prime}(t)\right)
$$

Case 5. If $y(t)$ is $[i i-g H]$-differentiable and $y_{g H}^{\prime}(t)$ and $y_{g H}^{\prime \prime}(t)$ are $[i-g H]$-differentiable,

$$
y_{i i . g H}^{\prime}(t)=\left(y_{3}^{\prime}(t), y_{2}^{\prime}(t), y_{1}^{\prime}(t)\right), y_{i i . g H}^{\prime \prime}(t)=\left(y_{3}^{\prime \prime}(t), y_{2}^{\prime \prime}(t), y_{1}^{\prime \prime}(t)\right), y_{i i . g H}^{\prime \prime \prime}(t)=\left(y_{3}^{\prime \prime \prime}(t), y_{2}^{\prime \prime \prime}(t), y_{1}^{\prime \prime \prime}(t)\right)
$$

Case 6. If $y(t)$ and $y_{g H}^{\prime \prime}(t)$ are $[i i-g H]$-differentiable and $y_{g H}^{\prime}(t)$ is $[i-g H]$-differentiable,

$$
y_{i i . g H}^{\prime}(t)=\left(y_{3}^{\prime}(t), y_{2}^{\prime}(t), y_{1}^{\prime}(t)\right), y_{i i . g H}^{\prime \prime}(t)=\left(y_{3}^{\prime \prime}(t), y_{2}^{\prime \prime}(t), y_{1}^{\prime \prime}(t)\right), y_{i . g H}^{\prime \prime \prime}(t)=\left(y_{1}^{\prime \prime \prime}(t), y_{2}^{\prime \prime \prime}(t), y_{3}^{\prime \prime \prime}(t)\right)
$$

Case 7. If $y(t)$ and $y_{g H}^{\prime}(t)$ are $[i i-g H]$-differentiable and $y_{g H}^{\prime \prime}(t)$ is $[i-g H]$-differentiable,

$$
y_{i i . g H}^{\prime}(t)=\left(y_{3}^{\prime}(t), y_{2}^{\prime}(t), y_{1}^{\prime}(t)\right), y_{i . g H}^{\prime \prime}(t)=\left(y_{1}^{\prime \prime}(t), y_{2}^{\prime \prime}(t), y_{3}^{\prime \prime}(t)\right), y_{i . g H}^{\prime \prime \prime}(t)=\left(y_{1}^{\prime \prime \prime}(t), y_{2}^{\prime \prime \prime}(t), y_{3}^{\prime \prime \prime}(t)\right)
$$

Case 8. If $y(t), y_{g H}^{\prime}(t)$ and $y_{g H}^{\prime \prime}(t)$ are $[i i-g H]$-differentiable,

$$
y_{i i . g H}^{\prime}(t)=\left(y_{3}^{\prime}(t), y_{2}^{\prime}(t), y_{1}^{\prime}(t)\right), y_{i . g H}^{\prime \prime}(t)=\left(y_{1}^{\prime \prime}(t), y_{2}^{\prime \prime}(t), y_{3}^{\prime \prime}(t)\right), y_{i i . g H}^{\prime \prime \prime}(t)=\left(y_{3}^{\prime \prime \prime}(t), y_{2}^{\prime \prime \prime}(t), y_{1}^{\prime \prime \prime}(t)\right)
$$

Definition 27 (See [9]) Let $y:(a, b) \rightarrow \mathbb{R}_{\mathscr{T}}$ is a triangular fuzzy-valued function and $t_{0} \in(a, b)$ then

$$
\int_{a}^{b} y(t) d t=\left(\int_{a}^{b} y_{1}(t) d t, \int_{a}^{b} y_{2}(t) d t, \int_{a}^{b} y_{3}(t) d t\right)
$$

Theorem 28 Let $y:[a, b] \rightarrow \mathbb{R}_{\mathscr{T}}$ be a triangular fuzzy function such that $y \in$ $\mathscr{C}_{g H}^{n}\left([a, b], \mathbb{R}_{\mathscr{T}}\right)$ with no switching points in $[a, b]$. Then for $j=1,2, \ldots, n$, there are the following different scenarios

i.

$$
\begin{aligned}
& y_{i . g H}^{(j-1)}(t+\Delta t)=y_{i \cdot g H}^{(j-1)}(t) \oplus \int_{t}^{t+\Delta t} y_{i . g H}^{(j)}(x) d x \\
& y_{i . g H}^{(j-1)}(t-\Delta t)=y_{i . g H}^{(j-1)}(t) \ominus \int_{t-\Delta t}^{t} y_{i . g H}^{(j)}(x) d x,
\end{aligned}
$$

ii.

$$
\begin{aligned}
& y_{i i . g H}^{(j-1)}(t+\Delta t)=y_{i i . g H}^{(j-1)}(t) \oplus \int_{t}^{t+\Delta t} y_{i i . g H}^{(j)}(x) d x \\
& y_{i i . g H}^{(j-1)}(t-\Delta t)=y_{i i . g H}^{(j-1)}(t) \ominus \int_{t-\Delta t}^{t} y_{i i . g H}^{(j)}(x) d x,
\end{aligned}
$$

iii.

$$
\begin{aligned}
& y_{i . g H}^{(j-1)}(t+\Delta t)=y_{i . g H}^{(j-1)}(t) \ominus(-1) \int_{t}^{t+\Delta t} y_{i i . g H}^{(j)}(x) d x \\
& y_{i . g H}^{(j-1)}(t-\Delta t)=y_{i . g H}^{(j-1)}(t) \oplus(-1) \int_{t-\Delta t}^{t} y_{i i . g H}^{(j)}(x) d x,
\end{aligned}
$$


iv.

$$
\begin{aligned}
& y_{\text {ii.gH }}^{(j-1)}(t+\Delta t)=y_{\text {ii.gH }}^{(j-1)}(t) \ominus(-1) \int_{t}^{t+\Delta t} y_{i . g H}^{(j)}(x) d x \\
& y_{\text {ii.gH }}^{(j-1)}(t-\Delta t)=y_{\text {ii.gH }}^{(j-1)}(t) \oplus(-1) \int_{t-\Delta t}^{t} y_{i . g H}^{(j)}(x) d x,
\end{aligned}
$$

Proof We have $y \in \mathscr{C}_{g H}^{n}\left([a, b], \mathbb{R}_{F}\right)$, therefore $f^{(j)}(t), j=0,1, \ldots, n$ are integrable. We will prove parts (i) and (iii); the other parts are similar, and we omit the details. By using Remark 26 and Definition 27 , we get

$$
\begin{aligned}
& y_{i . g H}^{(j-1)}(t) \oplus \int_{t}^{t+\Delta t} y_{i . g H}^{(j)}(x) d x \\
= & \left(y_{1}^{(j-1)}(t), y_{2}^{(j-1)}(t), y_{3}^{(j-1)}(t)\right) \oplus\left(\int_{t}^{t+\Delta t} y_{1}^{(j)}(x) d x, \int_{t}^{t+\Delta t} y_{2}^{(j)}(x) d x, \int_{t}^{t+\Delta t} y_{3}^{(j)}(x) d x\right) \\
= & \left(y_{1}^{(j-1)}(t), y_{2}^{(j-1)}(t), y_{3}^{(j-1)}(t)\right) \\
& \oplus\left(y_{1}^{(j-1)}(t+\Delta t)-y_{1}^{(j-1)}(t), y_{2}^{(j-1)}(t+\Delta t)-y_{2}^{(j-1)}(t), y_{3}^{(j-1)}(t+\Delta t)-y_{3}^{(j-1)}(t)\right) \\
= & \left(y_{1}^{(j-1)}(t+\Delta t), y_{2}^{(j-1)}(t+\Delta t), y_{3}^{(j-1)}(t+\Delta t)\right) .
\end{aligned}
$$

And

$$
\begin{aligned}
& y_{i . g H}^{(j-1)}(t) \ominus \int_{t-\Delta t}^{t} y_{i . g H}^{(j)}(x) d x \\
= & \left(y_{1}^{(j-1)}(t), y_{2}^{(j-1)}(t), y_{3}^{(j-1)}(t)\right) \ominus\left(\int_{t-\Delta t}^{t} y_{1}^{(j)}(x) d x, \int_{t-\Delta t}^{t} y_{2}^{(j)}(x) d x, \int_{t-\Delta t}^{t} y_{3}^{(j)}(x) d x\right) \\
= & \left(y_{1}^{(j-1)}(t), y_{2}^{(j-1)}(t), y_{3}^{(j-1)}(t)\right) \\
& \ominus\left(y_{1}^{(j-1)}(t)-y_{1}^{(j-1)}(t-\Delta t), y_{2}^{(j-1)}(t)-y_{2}^{(j-1)}(t-\Delta t), y_{3}^{(j-1)}(t)-y_{3}^{(j-1)}(t-\Delta t)\right) \\
= & \left(y_{1}^{(j-1)}(t-\Delta t), y_{2}^{(j-1)}(t-\Delta t), y_{3}^{(j-1)}(t-\Delta t)\right) .
\end{aligned}
$$

Now we want to prove case (iii). We have

$$
\begin{aligned}
& y_{i . g H}^{(j-1)}(t) \ominus(-1) \int_{t}^{t+\Delta t} y_{i i . g H}^{(j)}(x) d x \\
= & \left(y_{1}^{(j-1)}(t), y_{2}^{(j-1)}(t), y_{3}^{(j-1)}(t)\right) \ominus(-1)\left(\int_{t}^{t+\Delta t} y_{3}^{(j)}(x) d x, \int_{t}^{t+\Delta t} y_{2}^{(j)}(x) d x, \int_{t}^{t+\Delta t} y_{1}^{(j)}(x) d x\right) \\
= & \left(y_{1}^{(j-1)}(t), y_{2}^{(j-1)}(t), y_{3}^{(j-1)}(t)\right) \\
& \ominus(-1)\left(y_{3}^{(j-1)}(t+\Delta t)-y_{3}^{(j-1)}(t), y_{2}^{(j-1)}(t+\Delta t)-y_{2}^{(j-1)}(t), y_{1}^{(j-1)}(t+\Delta t)-y_{1}^{(j-1)}(t)\right) \\
= & \left(y_{1}^{(j-1)}(t), y_{2}^{(j-1)}(t), y_{3}^{(j-1)}(t)\right) \\
& \ominus\left(y_{1}^{(j-1)}(t)-y_{1}^{(j-1)}(t+\Delta t), y_{2}^{(j-1)}(t+\Delta t)-y_{2}^{(j-1)}(t), y_{3}^{(j-1)}(t)-y_{3}^{(j-1)}(t+\Delta t)\right) \\
= & \left(y_{1}^{(j-1)}(t+\Delta t), y_{2}^{(j-1)}(t+\Delta t), y_{3}^{(j-1)}(t+\Delta t)\right) .
\end{aligned}
$$


and

$$
\begin{aligned}
& y_{i . g H}^{(j-1)}(t) \oplus(-1) \int_{t-\Delta t}^{t} y_{i i . g H}^{(j)}(x) d x \\
= & \left(y_{1}^{(j-1)}(t), y_{2}^{(j-1)}(t), y_{3}^{(j-1)}(t)\right) \oplus(-1)\left(\int_{t-\Delta t}^{t} y_{3}^{(j)}(x) d x, \int_{t-\Delta t}^{t} y_{2}^{(j)}(x) d x, \int_{t-\Delta t}^{t} y_{1}^{(j)}(x) d x\right) \\
= & \left(y_{1}^{(j-1)}(t), y_{2}^{(j-1)}(t), y_{3}^{(j-1)}(t)\right) \\
& \oplus(-1)\left(y_{3}^{(j-1)}(t)-y_{3}^{(j-1)}(t-\Delta t), y_{2}^{(j-1)}(t)-y_{2}^{(j-1)}(t-\Delta t), y_{1}^{(j-1)}(t)-y_{1}^{(j-1)}(t-\Delta t)\right) \\
= & \left(y_{1}^{(j-1)}(t), y_{2}^{(j-1)}(t), y_{3}^{(j-1)}(t)\right) \\
& \oplus\left(y_{1}^{(j-1)}(t-\Delta t)-y_{1}^{(j-1)}(t), y_{2}^{(j-1)}(t-\Delta t)-y_{2}^{(j-1)}(t), y_{3}^{(j-1)}(t-\Delta t)-y_{3}^{(j-1)}(t)\right) \\
= & \left(y_{1}^{(j-1)}(t-\Delta t), y_{2}^{(j-1)}(t-\Delta t), y_{3}^{(j-1)}(t-\Delta t)\right),
\end{aligned}
$$

which proves this case.

Next, we are going to prove a crucial theorem to all the different cases in Remark 26, which will be used in the following sections. Actually, we will obtain four terms of the fuzzy Taylor's expansion about the point $t_{k}$ for $t_{k} \leq t$ and $t \leq t_{k}$ by considering different type of gH-differentiability for $y(t), y_{g H}^{\prime}(t)$ and $y_{g H}^{\prime \prime}(t)$.

Theorem 29 Let $\mathbb{T}=[a, b] \subset \mathbb{R}, y \in \mathscr{C}_{g H}^{4}([a, b], \mathbb{R} \mathscr{T})$. For $t, t \pm \Delta t \in \mathbb{T}$, we have

Case 1. If $y(t), y_{g H}^{\prime}(t)$ and $y_{g H}^{\prime \prime}(t)$ are $[i-g H]$-differentiable:

$$
\begin{aligned}
& y(t+\Delta t)=y(t) \oplus y_{i . g H}^{\prime}(t) \odot \Delta t \oplus y_{i . g H}^{\prime \prime}(t) \odot \frac{\Delta t^{2}}{2 !} \oplus y_{i . g H}^{\prime \prime \prime}(t) \odot \frac{\Delta t^{3}}{3 !} \diamond \mathscr{R}(t+\Delta t), \\
& y(t-\Delta t)=y(t) \ominus y_{i . g H}^{\prime}(t) \odot \Delta t \oplus y_{i . g H}^{\prime \prime}(t) \odot \frac{\Delta t^{2}}{2 !} \oplus(-1) y_{i i . g H}^{\prime \prime \prime}(t) \odot \frac{\Delta t^{3}}{3 !} \diamond \mathscr{R}(t-\Delta t)
\end{aligned}
$$

Case 2. If $y(t)$ and $y_{g H}^{\prime}(t)$ are $[i-g H]$-differentiable and $y_{g H}^{\prime \prime}(t)$ is $[i i-g H]$-differentiable:

$$
\begin{aligned}
& y(t+\Delta t)=y(t) \oplus y_{i . g H}^{\prime}(t) \odot \Delta t \oplus y_{i . g H}^{\prime \prime}(t) \odot \frac{\Delta t^{2}}{2 !} \ominus(-1) y_{i i . g H}^{\prime \prime \prime}(t) \odot \frac{\Delta t^{3}}{3 !} \diamond \mathscr{R}(t+\Delta t), \\
& y(t-\Delta t)=y(t) \ominus y_{i . g H}^{\prime}(t) \odot \Delta t \oplus y_{i . g H}^{\prime \prime}(t) \odot \frac{\Delta t^{2}}{2 !} \oplus(-1) y_{i i . g H}^{\prime \prime \prime}(t) \odot \frac{\Delta t^{3}}{3 !} \diamond \mathscr{R}(t-\Delta t)
\end{aligned}
$$

Case 3. If $y(t)$ and $y_{g H}^{\prime \prime}(t)$ are $[i-g H]$-differentiable and $y_{g H}^{\prime}(t)$ is $[i i-g H]$-differentiable:

$$
\begin{aligned}
& y(t+\Delta t)=y(t) \oplus y_{i . g H}^{\prime}(t) \odot \Delta t \ominus(-1) y_{i i . g H}^{\prime \prime}(t) \odot \frac{\Delta t^{2}}{2 !} \ominus(-1) y_{i i . g H}^{\prime \prime \prime}(t) \odot \frac{\Delta t^{3}}{3 !} \diamond \mathscr{R}(t+\Delta t), \\
& y(t-\Delta t)=y(t) \ominus y_{i . g H}^{\prime}(t) \odot \Delta t \ominus(-1) y_{i i . g H}^{\prime \prime}(t) \odot \frac{\Delta t^{2}}{2 !} \oplus(-1) y_{i i . g H}^{\prime \prime \prime}(t) \odot \frac{\Delta t^{3}}{3 !} \diamond \mathscr{R}(t-\Delta t) .
\end{aligned}
$$


Case 4. If $y(t)$ is $[i-g H]$-differentiable and $y_{g H}^{\prime}(t)$ and $y_{g H}^{\prime \prime}(t)$ are $[i i-g H]$-differentiable:

$$
\begin{aligned}
& y(t+\Delta t)=y(t) \oplus y_{i . g H}^{\prime}(t) \odot \Delta t \ominus(-1) y_{i i . g H}^{\prime \prime}(t) \odot \frac{\Delta t^{2}}{2 !} \oplus y_{i . g H}^{\prime \prime \prime}(t) \odot \frac{\Delta t^{3}}{3 !} \diamond \mathscr{R}(t+\Delta t), \\
& y(t-\Delta t)=y(t) \ominus y_{i . g H}^{\prime}(t) \odot \Delta t \ominus(-1) y_{i i . g H}^{\prime \prime}(t) \odot \frac{\Delta t^{2}}{2 !} \ominus y_{i . g H}^{\prime \prime \prime}(t) \odot \frac{\Delta t^{3}}{3 !} \diamond \mathscr{R}(t-\Delta t) .
\end{aligned}
$$

Case 5. If $y(t)$ is $[i i-g H]$-differentiable and $y_{g H}^{\prime}(t)$ and $y_{g H}^{\prime \prime}(t)$ are $[i-g H]$-differentiable:

$$
\begin{aligned}
& y(t+\Delta t)=y(t) \ominus(-1) y_{i i . g H}^{\prime}(t) \odot \Delta t \ominus(-1) y_{i i . g H}^{\prime \prime}(t) \odot \frac{\Delta t^{2}}{2 !} \ominus(-1) y_{i i . g H}^{\prime \prime \prime}(t) \odot \frac{\Delta t^{3}}{3 !} \diamond \mathscr{R}(t+\Delta t), \\
& y(t-\Delta t)=y(t) \oplus(-1) y_{i i . g H}^{\prime}(t) \odot \Delta t \ominus(-1) y_{i i . g H}^{\prime \prime}(t) \odot \frac{\Delta t^{2}}{2 !} \ominus(-1) y_{i i . g H}^{\prime \prime \prime}(t) \odot \frac{\Delta t^{3}}{3 !} \diamond \mathscr{R}(t-\Delta t) .
\end{aligned}
$$

Case 6. If $y(t)$ and $y_{g H}^{\prime \prime}(t)$ are $[i i-g H]$-differentiable and $y_{g H}^{\prime}(t)$ is $[i-g H]$-differentiable:

$$
\begin{aligned}
& y(t+\Delta t)=y(t) \ominus(-1) y_{i i . g H}^{\prime}(t) \odot \Delta t \ominus(-1) y_{i i . g H}^{\prime \prime}(t) \odot \frac{\Delta t^{2}}{2 !} \oplus y_{i . g H}^{\prime \prime \prime}(t) \odot \frac{\Delta t^{3}}{3 !} \diamond \mathscr{R}(t+\Delta t), \\
& y(t-\Delta t)=y(t) \oplus(-1) y_{i i . g H}^{\prime}(t) \odot \Delta t \ominus(-1) y_{i i . g H}^{\prime \prime}(t) \odot \frac{\Delta t^{2}}{2 !} \oplus y_{i . g H}^{\prime \prime \prime}(t) \odot \frac{\Delta t^{3}}{3 !} \diamond \mathscr{R}(t-\Delta t) .
\end{aligned}
$$

Case 7. If $y(t)$ and $y_{g H}^{\prime}(t)$ are $[i i-g H]$-differentiable and $y_{g H}^{\prime \prime}(t)$ is $[i-g H]$-differentiable:

$$
\begin{aligned}
& y(t+\Delta t)=y(t) \ominus(-1) y_{i i . g H}^{\prime}(t) \odot \Delta t \oplus y_{i . g H}^{\prime \prime}(t) \odot \frac{\Delta t^{2}}{2 !} \ominus(-1) y_{i . g H}^{\prime \prime \prime}(t) \odot \frac{\Delta t^{3}}{3 !} \diamond \mathscr{R}(t+\Delta t), \\
& y(t-\Delta t)=y(t) \oplus(-1) y_{i i . g H}^{\prime}(t) \odot \Delta t \oplus y_{i . g H}^{\prime \prime}(t) \odot \frac{\Delta t^{2}}{2 !} \ominus(-1) y_{i i . g H}^{\prime \prime \prime}(t) \odot \frac{\Delta t^{3}}{3 !} \diamond \mathscr{R}(t-\Delta t) .
\end{aligned}
$$

Case 8. If $y(t)$ and $y_{g H}^{\prime}(t)$ are $[i i-g H]$-differentiable and $y_{g H}^{\prime \prime}(t)$ is $[i-g H]$-differentiable:

$$
\begin{aligned}
& y(t+\Delta t)=y(t) \ominus(-1) y_{i i . g H}^{\prime}(t) \odot \Delta t \oplus y_{i . g H}^{\prime \prime}(t) \odot \frac{\Delta t^{2}}{2 !} \oplus y_{i . g H}^{\prime \prime \prime}(t) \odot \frac{\Delta t^{3}}{3 !} \diamond \mathscr{R}(t+\Delta t), \\
& y(t-\Delta t)=y(t) \oplus(-1) y_{i i . g H}^{\prime}(t) \odot \Delta t \oplus y_{i . g H}^{\prime \prime}(t) \odot \frac{\Delta t^{2}}{2 !} \oplus y_{i . g H}^{\prime \prime \prime}(t) \odot \frac{\Delta t^{3}}{3 !} \diamond \mathscr{R}(t-\Delta t) .
\end{aligned}
$$

where

$$
\mathscr{R}(t+\Delta t)=\int_{t}^{t+\Delta t}\left(\int_{t}^{\xi_{1}}\left(\int_{t}^{\xi_{2}}\left(\int_{t}^{\xi_{3}} y_{g H}^{(4)}\left(\xi_{4}\right) d \xi_{4}\right) d \xi_{3}\right) d \xi_{2}\right) d \xi_{1} .
$$

and

$$
\mathscr{R}(t-\Delta t)=\int_{t-\Delta t}^{t}\left(\int_{\xi_{1}}^{t}\left(\int_{\xi_{2}}^{t}\left(\int_{\xi_{3}}^{t} y_{g H}^{(4)}\left(\xi_{4}\right) d \xi_{4}\right) d \xi_{3}\right) d \xi_{2}\right) d \xi_{1}
$$

and $\diamond$ can be one of the $\oplus, \oplus(-1)$ or $\ominus(-1)$. 
Proof Since $y \in \mathscr{C}_{g H}^{4}\left([a, b], \mathbb{R}_{\mathscr{T}}\right)$ with no switching points, so $y_{g H}^{(i)}, i=0,1,2,3,4$ are integrable on $[a, b]$. We want to prove Case 1, therefore $y(t), y_{g H}^{\prime}(t)$ and $y_{g H}^{\prime \prime}(t)$ are $[i-g H]$-differentiable. According to Theorem 28, we can write

$$
y(t+\Delta t)=y(t) \oplus \int_{t}^{t+\Delta t} y_{i . g H}^{\prime}\left(\xi_{1}\right) d \xi_{1}
$$

and

$$
y_{i . g H}^{\prime}\left(\xi_{1}\right)=y_{i . g H}^{\prime}(t) \oplus \int_{t}^{\xi_{1}} y_{i . g H}^{\prime \prime}\left(\xi_{2}\right) d \xi_{2} .
$$

By integration from each side of equation (3), we conclude that

$$
\begin{aligned}
\int_{t}^{t+\Delta t} y_{i . g H}^{\prime}\left(\xi_{1}\right) d \xi_{1} & =\int_{t}^{t+\Delta t} y_{i . g H}^{\prime}(t) d \xi_{1} \oplus \int_{t}^{t+\Delta t}\left(\int_{t}^{\xi_{1}} y_{i . g H}^{\prime \prime}\left(\xi_{2}\right) d \xi_{2}\right) d \xi_{1} \\
& =y_{i . g H}^{\prime}(t) \odot \Delta t \oplus \int_{t}^{t+\Delta t}\left(\int_{t}^{\xi_{1}} y_{i . g H}^{\prime \prime}\left(\xi_{2}\right) d \xi_{2}\right) d \xi_{1} .
\end{aligned}
$$

By continuing this process

$$
y_{i . g H}^{\prime \prime}\left(\xi_{2}\right)=y_{i . g H}^{\prime \prime}(t) \oplus \int_{t}^{\xi_{2}} y_{i . g H}^{\prime \prime \prime}\left(\xi_{3}\right) d \xi_{3} .
$$

Applying the integral operator to $y_{i . g H}^{\prime \prime}\left(\xi_{2}\right)$, gives

$$
\int_{t}^{\xi_{1}} y_{i . g H}^{\prime \prime}\left(\xi_{2}\right) d \xi_{2}=y_{i . g H}^{\prime \prime}(t) \odot\left(\xi_{1}-t\right) \oplus \int_{t}^{\xi_{1}}\left(\int_{t}^{\xi_{2}} y_{i . g H}^{\prime \prime \prime}\left(\xi_{3}\right) d \xi_{3}\right) d \xi_{2}
$$

furthermore,

$\int_{t}^{t+\Delta t}\left(\int_{t}^{\xi_{1}} y_{i . g H}^{\prime \prime}\left(\xi_{2}\right) d \xi_{2}\right) d \xi_{1}=y_{i . g H}^{\prime \prime}(t) \odot \int_{t}^{t+\Delta t}\left(\xi_{1}-t\right) d \xi_{1} \oplus \int_{t}^{t+\Delta t}\left(\int_{t}^{\xi_{1}}\left(\int_{t}^{\xi_{2}} y_{i . g H}^{\prime \prime \prime}\left(\xi_{3}\right) d \xi_{3}\right) d \xi_{2}\right) d \xi_{1}$.

and

$$
y(t+\Delta t)=y(t) \oplus y_{i . g H}^{\prime}(t) \odot \Delta t \oplus y_{i . g H}^{\prime \prime}(t) \odot \frac{\Delta t^{2}}{2 !} \oplus \int_{t}^{t+\Delta t}\left(\int_{t}^{\xi_{1}}\left(\int_{t}^{\xi_{2}} y_{i . g H}^{\prime \prime \prime}\left(\xi_{3}\right) d \xi_{3}\right) d \xi_{2}\right) d \xi_{1} .
$$

With the similar manner,

$$
y(t+\Delta t)=y(t) \oplus y_{i . g H}^{\prime}(t) \odot \Delta t \oplus y_{i . g H}^{\prime \prime}(t) \odot \frac{\Delta t^{2}}{2 !} \oplus y_{i . g H}^{\prime \prime \prime}(t) \odot \frac{\Delta t^{3}}{3 !} \diamond \mathscr{R}(t+\Delta t),
$$

where

$$
\mathscr{R}(t+\Delta t)=\int_{t}^{t+\Delta t}\left(\int_{t}^{\xi_{1}}\left(\int_{t}^{\xi_{2}}\left(\int_{t}^{\xi_{3}} y_{g H}^{\prime \prime \prime \prime}\left(\xi_{4}\right) d \xi_{4}\right) d \xi_{3}\right) d \xi_{2}\right) d \xi_{1},
$$

Now consider $y(t)$ is a continues and $[i-g H]$-differentiable fuzzy function. By using Theorem 28 for $j=1$ we can write

$$
y(t-\Delta t)=y(t) \ominus \int_{t-\Delta t}^{t} y_{i . g H}^{\prime}\left(\xi_{1}\right) d \xi_{1}
$$


according to Theorem 28

$$
y_{i . g H}^{\prime}\left(\xi_{1}\right)=y_{i . g H}^{\prime}(t) \ominus \int_{\xi_{1}}^{t} y_{i . g H}^{\prime \prime}\left(\xi_{2}\right) d \xi_{2} .
$$

Therefore by integration of (4), we get that

$$
\begin{aligned}
\int_{t-\Delta t}^{t} y_{i . g H}^{\prime}\left(\xi_{1}\right) d \xi_{1} & =\int_{t-\Delta t}^{t} y_{i . g H}^{\prime}(t) d \xi_{1} \ominus \int_{t-\Delta t}^{t}\left(\int_{\xi_{1}}^{t} y_{i . g H}^{\prime \prime}\left(\xi_{2}\right) d \xi_{2}\right) d \xi_{1} \\
& =y_{i . g H}^{\prime}(t) \odot \Delta t \ominus \int_{t-\Delta t}^{t}\left(\int_{\xi_{1}}^{t} y_{i . g H}^{\prime \prime}\left(\xi_{2}\right) d \xi_{2}\right) d \xi_{1} .
\end{aligned}
$$

Therefore

$$
y(t-\Delta t)=y(t) \ominus y_{i . g H}^{\prime}(t) \odot \Delta t \oplus \int_{t-\Delta t}^{t}\left(\int_{\xi_{1}}^{t} y_{i . g H}^{\prime \prime}\left(\xi_{2}\right) d \xi_{2}\right) d \xi_{1} .
$$

But we have

$$
y_{i . g H}^{\prime \prime}\left(\xi_{2}\right)=y_{i . g H}^{\prime \prime}(t) \ominus \int_{\xi_{2}}^{t} y_{i . g H}^{\prime \prime \prime}\left(\xi_{3}\right) d \xi_{3}
$$

With repeated integrals we have

$$
\begin{aligned}
& \int_{\xi_{1}}^{t} y_{i . g H}^{\prime \prime}\left(\xi_{2}\right) d \xi_{2}=y_{i . g H}^{\prime \prime}(t) \odot\left(t-\xi_{1}\right) \ominus \int_{\xi_{1}}^{t}\left(\int_{\xi_{2}}^{t} y_{i . g H}^{\prime \prime \prime}\left(\xi_{3}\right) d \xi_{3}\right) d \xi_{2}, \quad \Rightarrow \\
& \int_{t-\Delta t}^{t}\left(\int_{\xi_{1}}^{t} y_{i . g H}^{\prime \prime}\left(\xi_{2}\right) d \xi_{2}\right) d \xi_{1}=y_{i . g H}^{\prime \prime}(t) \odot \int_{t-\Delta t}^{t}\left(t-\xi_{1}\right) d \xi_{1} \ominus \int_{t-\Delta t}^{t}\left(\int_{\xi_{1}}^{t}\left(\int_{\xi_{2}}^{t} y_{i . g H}^{\prime \prime \prime}\left(\xi_{3}\right) d \xi_{3}\right) d \xi_{2}\right) d \xi_{1} .
\end{aligned}
$$

In this case we can conclude

$$
y(t-\Delta t)=y(t) \ominus y_{i . g H}^{\prime}(t) \odot \Delta t \oplus y_{i \cdot g H}^{\prime \prime}(t) \odot \frac{\Delta t^{2}}{2 !} \ominus \int_{t-\Delta t}^{t}\left(\int_{\xi_{1}}^{t}\left(\int_{\xi_{2}}^{t} y_{i . g H}^{\prime \prime \prime}\left(\xi_{3}\right) d \xi_{3}\right) d \xi_{2}\right) d \xi_{1} .
$$

In the same way, the other cases outlined in the theorem are also proven using Theorem 28 .

Definition 210 (See [5]) The first generalized Hukuhara partial derivative ( [gH-p]-derivative for short) of a fuzzy-valued function $u(x, t): \mathbb{D} \subseteq \mathbb{R}^{2} \rightarrow \mathbb{R}_{F}$ at $\left(x_{0}, t_{0}\right)$ with respect to variables $x$ is defined by

$$
\partial_{x_{g H}} u\left(x_{0}, t_{0}\right)=\lim _{h \rightarrow 0} \frac{u\left(x_{0}+h, t_{0}\right) \ominus_{g H} u\left(x_{0}, t_{0}\right)}{h},
$$

A triangular fuzzy function $u(x, t): \mathbb{D} \subseteq \mathbb{R}^{2} \rightarrow \mathbb{R}_{\mathscr{T}}$, without any switching point on $\mathbb{D}$ is called

- $[(i)-p]$-differentiable w.r.t. $x$ at $\left(x_{0}, t_{0}\right)$ if

$$
u_{x_{i . g H}}\left(x_{0}, t_{0}\right)=\left(u_{1_{x}}\left(x_{0}, t_{0}\right), u_{2_{x}}\left(x_{0}, t_{0}\right), u_{3_{x}}\left(x_{0}, t_{0}\right)\right)
$$


$-[(i i)-p]$-differentiable w.r.t. $x$ at $\left(x_{0}, t_{0}\right)$ if

$$
u_{x_{i . . g H}}\left(x_{0}, t_{0}\right)=\left(u_{3_{x}}\left(x_{0}, t_{0}\right), u_{2_{x}}\left(x_{0}, t_{0}\right), u_{1_{x}}\left(x_{0}, t_{0}\right)\right) .
$$

Moreover, if $u_{x_{g H}}(x, t)$ is $[g H-p]$-differentiable at $\left(x_{0}, t_{0}\right)$ with respect to $x$ without any switching point on $\mathbb{D}$ and

- if the type of $[g H-p]$-differentiability of both $u(x, t)$ and $u_{x_{g H}}(x, t)$ are the same, then $u_{x_{g H}}(x, t)$ is $[(i)-p]$-differentiable w.r.t $x$ and

$$
u_{x x_{i, g H}}\left(x_{0}, t_{0}\right)=\left(u_{1_{x x}}\left(x_{0}, t_{0}\right), u_{2_{x x}}\left(x_{0}, t_{0}\right), u_{3_{x x}}\left(x_{0}, t_{0}\right)\right) .
$$

- if the type of [gH-p]-differentiability $u(x, t)$ and $u_{x_{g H}}(x, t)$ are different, therefore $u_{x_{g H}}(x, t)$ is $[(i i)-p]$-differentiable w.r.t $x$ and

$$
u_{x x_{i i . g H}}\left(x_{0}, t_{0}\right)=\left(u_{3_{x x}}\left(x_{0}, t_{0}\right), u_{2 x x}\left(x_{0}, t_{0}\right), u_{1_{x x}}\left(x_{0}, t_{0}\right)\right) .
$$

\section{Finite Difference Methods}

Our goal here is to describe the fundamentals of the fuzzy finite difference method. To accomplish this, we will first show you how to obtain the finite difference formula for the first and second derivatives of a triangular fuzzy function $y(t)$.

Now, we describe the essential details of finite difference methods. First, we select an integer $N>0$ and divide the interval $[a, b]$ into $(N+1)$ equal subintervals whose endpoints are the mesh points $t_{i}=a+i \Delta t$, for $i=0,1, \ldots, N+1$, where $\Delta t=\frac{b-a}{N+1}$ so $t_{i+1}-t_{i}=\Delta t$. Let $y(t) \in \mathscr{C}_{g H}^{4}\left([a, b], \mathbb{R}_{\mathscr{T}}\right)$, so based on the different types of differentiability that mentioned in Remark 26, the first and second gH-derivative of this fuzzy function can be approximated by fuzzy finite difference method as follows

Case 3.1 Consider $y(t)$ and $y_{g H}^{\prime}(t)$ are $[i . g H]$-differentiable and $y_{g H}^{\prime \prime}(t)$ is $[i-$ $g H]$-differentiable or $[i i-g H]$-differentiable.

- The first fuzzy forward difference.

First Consider $y_{g H}^{\prime \prime}(t)$ is $[i-g H]$-differentiable. Hence by using the Case 1 in Theorem 29, we have

$y(t+\Delta t)=y(t) \oplus y_{i . g H}^{\prime}(t) \odot \Delta t \oplus y_{i . g H}^{\prime \prime}(t) \odot \frac{\Delta t^{2}}{2 !} \oplus y_{i . g H}^{\prime \prime \prime}(t) \odot \frac{\Delta t^{3}}{3 !} \diamond \mathscr{R}(t+\Delta t \chi \zeta 5)$

solve for $y_{i . g H}^{\prime}(t)$ yields

$$
y_{i . g H}^{\prime}(t)=\frac{y(t+\Delta t) \ominus y(t)}{\Delta t} \ominus \frac{\Delta t}{2} y^{\prime \prime}(t) \ominus \frac{\Delta t^{2}}{3 !} y^{\prime \prime \prime}(t) \ominus \cdots,
$$

By using the fuzzy mean value theorem in [5], for all $i=0,1, \ldots, N$, there are $\xi^{+} \in(t, t+\Delta t)$ such that

$$
y_{i . g H}^{\prime}(t)=\frac{y(t+\Delta t) \ominus y(t)}{\Delta t} \ominus \frac{\Delta t}{2} y^{\prime \prime}\left(\xi^{+}\right),
$$


Or

$$
y_{i . g H}^{\prime}(t) \ominus \frac{y(t+\Delta t) \ominus y(t)}{\Delta t}=\ominus \frac{\Delta t}{2} y^{\prime \prime}\left(\xi^{+}\right)
$$

where the term $\frac{\Delta t}{2} y^{\prime \prime}\left(\xi^{+}\right)$is called truncation error of the forward fuzzy finite difference approximation. Moreover, the properties of the Hausdorff distance [20] are implied that

$D\left(y_{i . g H}^{\prime}(t), \frac{y(t+\Delta t) \ominus y(t)}{\Delta t} \ominus \frac{\Delta t}{2} y^{\prime \prime}\left(\xi^{+}\right)\right) \leq D\left(y_{i . g H}^{\prime}(t), \frac{y(t+\Delta t) \ominus y(t)}{\Delta t}\right)+D\left(0, \ominus \frac{\Delta t}{2} y^{\prime \prime}\left(\xi^{+}\right)\right) \rightarrow 0$,

as $\Delta t \rightarrow 0$. Therefore, $\Delta t$ should be sufficiently small to get a good approximation. Finally, for sufficiently small $\Delta t$, the first forward fuzzy finite difference approximation of $y_{i . g H}^{\prime}(t)$ is

$$
y_{i . g H}^{\prime}(t) \approx \frac{y(t+\Delta t) \ominus y(t)}{\Delta t} .
$$

- The first fuzzy backward difference.

To obtain the backward fuzzy finite difference formula, using Theorem 29 (case 1) we can write

$\left.y(t-\Delta t)=y(t) \ominus y_{i . g H}^{\prime}(t) \odot \Delta t \oplus y_{i . g H}^{\prime \prime}(t) \odot \frac{\Delta t^{2}}{2 !} \ominus y_{i . g H}^{\prime \prime \prime}(t) \odot \frac{\Delta t^{3}}{3 !} \diamond \mathscr{R}(t-\Delta t\rangle 7\right)$

Rearranging equation (7) gives

$$
\frac{y(t) \ominus y(t-\Delta t)}{\Delta t}=y_{i . g H}^{\prime}(t) \ominus y_{i . g H}^{\prime \prime}(t) \odot \frac{\Delta t}{2 !} \oplus y_{i . g H}^{\prime \prime \prime}(t) \odot \frac{\Delta t^{2}}{3 !} \diamond \ldots .
$$

For having a more useful approximation value for $y_{i . g H}^{\prime}(t)$, by using the fuzzy mean value theorem in [5], there are $\xi^{-} \in(t-\Delta t, t)$ such that

$$
\frac{y(t) \ominus y(t-\Delta t)}{\Delta t}=y_{i . g H}^{\prime}(t) \ominus y_{i . g H}^{\prime \prime}\left(\xi^{-}\right) \odot \frac{\Delta t}{2 !}
$$

So by considering $\Delta t$ is small enough, the approximation value obtained for the first-order gH-derivative is equal to

$$
y_{i . g H}^{\prime}(t) \approx \frac{y(t) \ominus y(t-\Delta t)}{\Delta t} .
$$

- The first fuzzy central difference.

We using Hukuhara subtract Eq. (5) from Eq. (7) and divide by $2 \Delta t$, then we obtain

$$
\frac{y(t+\Delta t) \ominus y(t-\Delta t)}{2 \Delta t}=y_{i . g H}^{\prime}(t) \oplus \frac{\Delta t^{2}}{12} y_{i . g H}^{\prime \prime \prime}(\xi) .
$$

On the other hand, given the Hausdorff distance properties, it can be seen that

$D\left(\frac{y(t+\Delta t) \ominus y(t-\Delta t)}{2 \Delta t}, y_{i . g H}^{\prime}(t) \oplus \frac{\Delta t^{2}}{12} y_{i . g H}^{\prime \prime \prime}(\xi)\right) \leq D\left(\frac{y(t+\Delta t) \ominus y(t-\Delta t)}{2 \Delta t}, y_{i . g H}^{\prime}(t)\right)+D\left(0, \frac{\Delta t^{2}}{12} y_{i . g H}^{\prime \prime \prime}(\xi)\right) \rightarrow 0$, 
When $\Delta t \rightarrow 0$, we have following equation

$$
y_{i . g H}^{\prime}(t) \approx \frac{y(t+\Delta t) \ominus y(t-\Delta t)}{2 \Delta t},
$$

is the first fuzzy central difference approximation of $y_{i . g H}^{\prime}(t)$.

- The second-order fuzzy central difference.

To obtain an appropriate approximation for the second-order derivative of the fuzzy function $y(t),(5)$ are added to $(7)$, then the equations are rearranged and divided by $\Delta t^{2}$

$$
\frac{y(t+\Delta t) \oplus y(t-\Delta t) \ominus 2 y(t)}{\Delta t^{2}}=y_{i . g H}^{\prime \prime}(t) \diamond \frac{1}{\Delta t^{2}}(\mathscr{R}(t+\Delta t) \oplus \mathscr{R}(t-\Delta t)) .
$$

Beside

$$
\begin{aligned}
& D\left(\frac{y(t+\Delta t) \oplus y(t-\Delta t) \ominus 2 y(t)}{\Delta t^{2}}, y_{i . g H}^{\prime \prime}(t) \diamond \frac{1}{\Delta t^{2}}(\mathscr{R}(t+\Delta t) \oplus \mathscr{R}(t-\Delta t))\right) \\
& \leq D\left(\frac{y(t+\Delta t) \oplus y(t-\Delta t) \ominus 2 y(t)}{\Delta t^{2}}, y_{i . g H}^{\prime \prime}(t)\right)+D\left(0, \frac{1}{\Delta t^{2}}(\mathscr{R}(t+\Delta t) \oplus \mathscr{R}(t-\Delta t))\right),
\end{aligned}
$$

but by the definition of $\mathscr{R}(t+\Delta t)$ and $\mathscr{R}(t-\Delta t)$ when $\Delta t \rightarrow 0$

$$
D\left(\frac{y(t+\Delta t) \oplus y(t-\Delta t) \ominus 2 y(t)}{\Delta t^{2}}, y_{i . g H}^{\prime \prime}(t)\right) \rightarrow 0, \quad D\left(0, \frac{1}{\Delta t^{2}}(\mathscr{R}(t+\Delta t) \oplus \mathscr{R}(t-\Delta t))\right) \rightarrow 0
$$

Hence, for $\Delta t$ sufficiently small, the appropriate approximation obtained for the second-order derivative $y_{i . g H}^{\prime \prime}\left(t_{i}\right)$ is equal to

$$
y_{i . g H}^{\prime \prime}(t) \approx \frac{y(t+\Delta t) \oplus y(t-\Delta t) \ominus 2 y(t)}{\Delta t^{2}} .
$$

Now, if $y_{g H}^{\prime \prime}(t)$ is $[i i-g H]$-differentiable we obtain the similar approximation value for $y_{i . g H}^{\prime}(t)$ and $y_{i . g H}^{\prime \prime}(t)$ and there is no need to repeat the process of obtaining these approximate values. Accordingly, the type of gH-differentiability of the second-order derivative has no effect on the obtained values, and these approximation values all depend on the fuzzy function $y(t)$ and its first-order derivative $\left.y_{[} g H\right]$.

Here, for the first and second $\mathrm{gH}$-derivatives, we present the relevant fuzzy finite difference formulas by considering the type of $\mathrm{gH}$-differentiability. We will not elaborate on the proof in these cases since it is the same as Case 3.1.

Case 3.2. If $y(t)$ is $[i-g H]$-differentiable and $y_{g H}^{\prime}(t)$ is $[i i-g H]$-differentiable. In this case $y_{g H}^{\prime \prime}(t)$ can be $[i-g H]$-differentiable or $[i i-g H]$-differentiable.

- The first fuzzy forward difference.

$$
y_{i . g H}^{\prime}(t) \approx \frac{y(t+\Delta t) \ominus y(t)}{\Delta t}
$$


- The first fuzzy backward difference.

$$
y_{i i . g H}^{\prime}(t) \approx \frac{y(t) \ominus y(t-\Delta t)}{\Delta t}
$$

- The first fuzzy central difference.

$$
y_{i . g H}^{\prime}(t) \approx \frac{y(t+\Delta t) \ominus y(t-\Delta t)}{2 \Delta t}
$$

- The second-order fuzzy central difference.

$$
y_{i i . g H}^{\prime \prime}(t) \approx \frac{-(2 y(t) \ominus y(t-\Delta t) \ominus y(t+\Delta t))}{\Delta t^{2}}
$$

Case 3.3. consider $y(t)$ is $[i i-g H]$-differentiable and $y_{g H}^{\prime}(t)$ is $[i-g H]$-differentiable.

In this case $y_{g H}^{\prime \prime}(t)$ may be $[i-g H]$-differentiable or $[i i-g H]$-differentiable.

- The first fuzzy forward difference.

$$
y_{\text {ii.gH }}^{\prime}(t) \approx \frac{-1(y(t) \ominus y(t+\Delta t))}{\Delta t}
$$

- The first fuzzy backward difference.

$$
y_{\text {ii.gH }}^{\prime}(t) \approx \frac{-1(y(t-\Delta t) \ominus y(t))}{\Delta t}
$$

- The first fuzzy central difference.

$$
y_{i i . g H}^{\prime}(t) \approx \frac{-1(y(t-\Delta t) \ominus y(t+\Delta t))}{2 \Delta t}
$$

- The second-order fuzzy central difference.

$$
y_{i i . g H}^{\prime \prime}(t) \approx \frac{-1(2 y(t) \ominus y(t+\Delta t) \ominus y(t-\Delta t))}{\Delta t^{2}}
$$

Case 3.4. Let $y(t)$ and $y_{g H}^{\prime}(t)$ are $[i i-g H]$-differentiable. $y_{g H}^{\prime \prime}(t)$ can be $[i-$ $g H]$-differentiable or $[i i-g H]$-differentiable, the final formulas are obtained same.

- The first fuzzy forward difference.

$$
y_{\text {ii.gH }}^{\prime}(t) \approx \frac{-1(y(t) \ominus y(t+\Delta t)}{\Delta t}
$$

- The first fuzzy backward difference.

$$
y_{\text {ii.gH }}^{\prime}(t) \approx \frac{-1(y(t-\Delta t) \ominus y(t))}{\Delta t}
$$

- The first fuzzy central difference.

$$
y_{i i . g H}^{\prime}(t) \approx \frac{-1(y(t-\Delta t) \ominus y(t+\Delta t))}{2 \Delta t}
$$

- The second-order fuzzy central difference.

$$
y_{i . g H}^{\prime \prime}(t) \approx \frac{(y(t+\Delta t) \oplus y(t-\Delta t) \ominus 2 y(t))}{\Delta t^{2}}
$$


4 The Non-homogeneous Fuzzy Heat Equation

In mathematical physics, motion or transport of particles, i.e., ions, molecules, etc., from higher concentration to lower concentration is modeled by the diffusion equation with appropriate boundary and initial conditions. Heat conduction in a rod is a prototypical diffusion equation. Consider a uniform rod of length $L$ which is insulated everywhere except at its two ends and the temperature is transmitted non-uniformly from beginning to end. This temperature denotes by $u(x, t)$ and $x$ is a coordinate in space, $t$ represents time. Measuring the temperature is an uncertain problem, and this vagueness maybe appears in the initial and boundary conditions. Suppose the temperature at the ends are kept at a fixed fuzzy temperature of $u(0, t)$ and $u(L, t)$, respectively. The problem is to find the future temperature along the rod by considering the given fuzzy initial temperature $u(x, 0)$. In this case, the above problem is formulated as the following fuzzy non-homogeneous initial-boundary-value heat equation

$$
\begin{cases}u_{t_{g H}}(x, t)=u_{x x_{g H}}(x, t) \oplus F(x, t), & x \in[0, L], t \in[0, T] \\ u(x, 0)=f(x), & x \in[0, L], \\ u(0, t)=g(t), & t>0, \\ u(L, t)=h(t) & t>0 .\end{cases}
$$

Where $f(x), g(t), h(t)$ and $F(x, t)$ are triangular fuzzy functions such that $f(x), g(t), h(x)$ and $F(x, t) \in \mathscr{C}_{g H}^{4}\left([0, L] \times[0, T], \mathbb{R}_{\mathscr{T}}\right)$, and

$$
\begin{array}{ll}
f(x)=\left(f_{1}(x), f_{2}(x), f_{3}(x)\right), \quad g(t)=\left(g_{1}(t), g_{2}(t), g_{3}(t)\right) \\
h(t)=\left(h_{1}(t), h_{2}(t), h_{3}(t)\right), \quad F(x, t)=\left(F_{1}(x, t), F_{2}(x, t), F_{3}(x, t)\right),
\end{array}
$$

This equation has a unique solution in different states of [gH-p]-differentiatiability [5] and the main purpose of this section is to obtain an approximate fuzzy solution for the fuzzy heat equation using the fuzzy finite difference method. Suppose that $u(x, t)$ is the exact fuzzy solution of equation (8) provided that the types of $[g H-p]$-differentiability with respect to $x$ and $t$ are the same. The basic idea is to replace all the derivatives in equation (8) by corresponding difference approximation.

Let $u(x, t) \in \mathscr{C}_{g H}^{4}\left([0, L] \times[0, T], \mathbb{R}_{\mathscr{T}}\right)$, by considering the type of $[g H-p]$-differentiability, the following different situations will be happen

Case 1. Let $u(x, t)$ is $[(i)-p]$-differentiable with respect to $t$ and $u_{x_{g H}}(x, t)$ is a $[(i)-p]$-differentiable fuzzy function with respect to $x$. In this case, the heat equation will be as follows

$$
u_{t_{i . g H}}(x, t)=u_{x x_{i \cdot g H}}(x, t) \oplus F(x, t) .
$$

- Forward Difference in time: Since $u(x, t)$ is $[(i)-p]$-differentiable with respect to $t$, then different cases 1,2,3 and 4 in Theorem 29 can be used, 
in which

$$
u(x, t+\Delta t)=u(x, t) \oplus u_{t_{i . g H}}(x, t) \odot \Delta t \oplus u_{t t_{i . g H}}(x, t) \odot \frac{\Delta t^{2}}{2 !} \oplus \ldots
$$

Therefore according to Section 3 we obtain

$$
u_{t_{i, g H}}(x, t) \approx \frac{u(x, t+\Delta t) \ominus u(x, t)}{\Delta t}
$$

- Central Differences in Space: Due to the fact that $u_{x_{g H}}(x, t)$ is a $[(i)-$ $p$ ]-differentiable function, all cases 1, 2, 7 and 8 which are expressed in Theorem 29, can be used. So let's take case 1

$$
\begin{aligned}
u(x+\Delta x, t) & =u(x, t) \oplus u_{x_{i \cdot g H}}(x, t) \odot \Delta x \oplus u_{x x_{i \cdot g H}}(x, t) \odot \frac{\Delta x^{2}}{2 !} \oplus u_{x x x_{i . g H}}(x, t) \\
& \odot \frac{\Delta x^{3}}{3 !} \diamond \mathscr{R}(x+\Delta x, t), \\
u(x-\Delta x, t) & =u(x, t) \ominus u_{x_{i \cdot g H}}(x, t) \odot \Delta x \oplus u_{x x_{i \cdot g H}}(x, t) \odot \frac{\Delta x^{2}}{2 !} \ominus u_{x x x_{i \cdot g H}}(x, t) \\
& \odot \frac{\Delta x^{3}}{3 !} \diamond \mathscr{R}(x+\Delta x, t) .
\end{aligned}
$$

Adding and re-arranging:

$$
u_{x x_{i, g H}}(x, t) \approx \frac{u(x+\Delta x, t) \ominus 2 u(x, t) \oplus u(x-\Delta x, t)}{\Delta x^{2}} .
$$

Now substitute equations (11) and (13) into the main equation (9), accordingly

$$
\frac{u(x, t+\Delta t) \ominus u(x, t)}{\Delta t}=\frac{u(x+\Delta x, t) \ominus 2 u(x, t) \oplus u(x-\Delta x, t)}{\Delta x^{2}} \oplus F(x, t),
$$

and

$u(x, t+\Delta t)=u(x, t) \oplus\left(\frac{\Delta t}{\Delta x^{2}}\right)(u(x+\Delta x, t) \ominus 2 u(x, t) \oplus u(x-\Delta x, t)) \oplus \Delta t F(x, t)$.

To obtain an approximation solution for equation (9) using the fuzzy finite difference method, we must divide the domain $[0, L] \times[0, T]$ into a set of mesh points. Here, we subdivide the domain $[0, L] \times[0, T]$ into $N_{x}+1$ and $N_{t}+1$ equally mesh points

$$
\begin{aligned}
x_{k} & =k \Delta x, \quad k=0, \ldots, N_{x}, \\
t_{n} & =n \Delta t, \quad n=0, \ldots, N_{t} .
\end{aligned}
$$

Now, consider $U_{k}^{n}$ denotes the mesh function that approximates $u\left(x_{k}, t_{n}\right)$ for $k=0, \ldots, N_{x}$ and $n=0, \ldots, N_{t}$. By putting mesh point $\left(x_{k}, t_{n}\right)$ into equation (9), the following formula is obtained

$$
U_{k}^{n+1}=U_{k}^{n} \oplus \mu\left(U_{k+1}^{n} \ominus 2 U_{k}^{n} \oplus U_{k-1}^{n}\right) \oplus \Delta t F_{k}^{n},
$$

where $\mu=\frac{\Delta t}{\Delta x^{2}}$. 
- Truncation error: Now, we want to investigate the truncation error of the scheme (14). The truncation error, $T(x, t)$, is the difference between two side of equation when the exact solution $u\left(x_{k}, t_{n}\right)$ is replaced with the approximation value $U_{k}^{n}$, hence

$$
T(x, t):=\frac{\Delta_{+t} u(x, t)}{\Delta t} \ominus \frac{\delta_{x}^{2} u(x, t)}{\Delta x^{2}},
$$

where

$$
\begin{aligned}
\Delta_{+t} u(x, t) & :=u(x, t+\Delta t) \ominus u(x, t) \\
\delta_{x}^{2} u(x, t) & :=u(x+\Delta x, t) \ominus 2 u(x, t) \oplus u(x-\Delta x, t)
\end{aligned}
$$

By using the fuzzy mean value theorem in [5], there is $\eta \in(t, t+\Delta t)$ such that equation (10) can be written as follows

$$
u(x, t+\Delta t) \ominus u(x, t)=u_{t_{i . g H}}(x, t) \odot \Delta t \oplus u_{t t_{i . g H}}(x, \eta) \odot \frac{\Delta t^{2}}{2 !},
$$

we obtain

$$
\Delta_{+t} u(x, t)=u_{t_{i . g H}}(x, t) \odot \Delta t \oplus u_{t t_{i . g H}}(x, \eta) \odot \frac{\Delta t^{2}}{2 !},
$$

On the other hand, equations (12) conclude that

$$
\delta_{x}^{2} u(x, t)=u_{x x_{i, g H}} \odot \Delta x^{2} \oplus(\mathscr{R}(x+\Delta x, t) \diamond \mathscr{R}(x-\Delta x, t)) .
$$

So

$$
\begin{aligned}
T(x, t) & =\left(u_{t_{i . g H}} \ominus u_{x x_{i . g H}}\right) \oplus\left(\frac{1}{2} u_{t t_{i . g H}}(x, \eta) \Delta t \ominus \frac{1}{\Delta x^{2}}(\mathscr{R}(x+\Delta x, t) \diamond \mathscr{R}(x-\Delta x, t))\right) \\
& =\frac{1}{2} u_{t t_{i . g H}}(x, \eta) \Delta t \ominus \frac{1}{\Delta x^{2}}(\mathscr{R}(x+\Delta x, t) \diamond \mathscr{R}(x-\Delta x, t)) .
\end{aligned}
$$

Since $u(x, t) \in \mathscr{C}_{g H}^{4}\left([0, L] \times[0, T], \mathbb{R}_{\mathscr{T}}\right)$, we can consider

$$
D\left(u_{t t_{i g H}}, 0\right) \leq M_{t t}, \quad D(\mathscr{R}(x+\Delta x, t) \diamond \mathscr{R}(x-\Delta x, t), 0) \leq M_{\mathscr{R}} .
$$

Then by the help of Hausdorff distance properties [20], we get the following result

$$
\begin{aligned}
D(T(x, t), 0) & \leq \frac{1}{2} M_{t t} \Delta t+\frac{1}{\Delta x^{2}} M_{\mathscr{R}} \\
& \leq \frac{1}{2} \Delta t\left(M_{t t}+\frac{\mu}{2} M_{\mathscr{R}}\right)
\end{aligned}
$$

So

$$
T(x, t) \rightarrow 0, \quad \text { as } \quad \Delta t \rightarrow 0, \forall(x, t) \in[0,1] \times[0, T] .
$$


- The convergence of method: To check the convergence of the given method, suppose fixed point $\left(x^{*}, t^{*}\right)$ in domain $[0, L] \times[0, T]$. We say that the method is convergent if $x_{k} \rightarrow x^{*}, \quad t_{n} \rightarrow t^{*}$ implies that

$$
U_{k}^{n} \rightarrow u\left(x^{*}, t^{*}\right)
$$

Let $e_{k}^{n}:=U_{k}^{n} \ominus u\left(x_{k}, t_{n}\right)$ be the error function for finite difference method in this case. Putting the error function in equation (14) and using Proposition 24 results to

$$
\begin{aligned}
e_{k}^{n+1} & =e_{k}^{n} \oplus \mu\left(e_{k+1}^{n} \ominus 2 e_{k}^{n} \oplus e_{k-1}^{n}\right) \ominus T_{k}^{n} \Delta t \\
& =(1-2 \mu) e_{k}^{n} \oplus \mu e_{k+1}^{n} \oplus \mu e_{k-1}^{n} \ominus T_{k}^{n} \Delta t,
\end{aligned}
$$

where $T_{k}^{n}:=T\left(x_{k}, t_{n}\right)$. Now, if $\mu \leq \frac{1}{2}$ the coefficient of the three terms of $e^{n}$ on the right-hand side of the above equation will be positive, and the result will be unity. Let us to consider

$$
\left.E^{n}:=\max \left\{D\left(e_{k}^{n}, 0\right), \quad k=0,1, \ldots, N_{x}\right)\right\} .
$$

So

$$
D\left(e_{k}^{n+1}, 0\right) \leq E^{n}+D\left(T_{k}^{n}, 0\right) \Delta t .
$$

Since this inequality holds for all values of $k$, then

$$
E^{n+1} \leq E^{n}+D\left(T_{k}^{n}, 0\right) \Delta t
$$

On the other hand, by definition of $e_{k}^{n}$ we know that $E^{0}=0$. In this case $E^{n} \leq n D\left(T_{k}^{n}, 0\right) \Delta t$, which is achieved with a simple induction. Therefore form (16) we obtain

$$
\begin{aligned}
E^{n} & \leq \frac{1}{2} \Delta t\left(M_{t t}+\frac{\mu}{2} M_{\mathscr{R}}\right) T \\
& \rightarrow 0 \text { as } \Delta t \rightarrow 0 .
\end{aligned}
$$

In fact we showed that the approximate solution $U_{k}^{n}$ obtained by finite difference method (14) converge to the exact solution $u\left(x_{k}, t_{n}\right)$ provided that $\mu \leq \frac{1}{2}$ for sufficiently large value of $N_{t}$, besides $D\left(u_{x x}, 0\right) \leq M_{x x}$ and $D(\mathscr{R}(x+\Delta x, t) \diamond \mathscr{R}(x-\Delta x, t), 0) \leq M_{\mathscr{R}}$.

The following algorithm summarizes the proposed fuzzy finite difference.

\section{Algorithm 41}

1. Choose $N_{t}$ and $N_{x}$ such that $\frac{\Delta t}{(\Delta x)^{2}} \leq \frac{1}{2}$.

2. Compute $U_{k}^{0}=\left(f_{1}\left(x_{k}\right), f_{2}\left(x_{k}\right), f_{3}\left(x_{k}\right)\right)$ for $k=0,1, \ldots, N_{x}$.

3. For $n=0,1, \ldots, N_{t}$ :

i. apply $U_{k}^{n+1}=U_{k}^{n} \oplus \mu\left(U_{k+1}^{n} \ominus 2 U_{k}^{n} \oplus U_{k-1}^{n}\right) \oplus \Delta t F_{k}^{n}$ for all $k=1, \ldots, N_{x}-1$.

ii. set the boundary value $U_{0}^{n+1}=\left(g_{1}\left(t_{n+1}\right), g_{2}\left(t_{n+1}\right), g_{3}\left(t_{n+1}\right)\right)$. 
iii. set the boundary value $U_{N_{x}}^{n+1}=\left(h_{1}\left(t_{n+1}\right), h_{2}\left(t_{n+1}\right), h_{3}\left(t_{n+1}\right)\right)$.

In the following, we will briefly consider the other case of the $[g H-p]$-differentiability for equation (8). The whole process of proof for the following situations is the same as in case 1, so we will not go into details and we just express the algorithm.

Case 2. Consider the following fuzzy heat equation

$$
u_{t_{i i . g H}}(x, t)=u_{x x_{i i . g H}}(x, t) \oplus F(x, t)
$$

in this equation $u(x, t)$ is $[(i i)-p]$-differentiable with respect to $t$ and $u_{x_{g H}}(x, t)$ is a $[(i i)-p]$-differentiable fuzzy function with respect to $x$.

Algorithm 42

1. Choose $N_{t}$ and $N_{x}$ such that $\frac{\Delta t}{(\Delta x)^{2}} \leq \frac{1}{2}$.

2. Compute $U_{k}^{0}=\left(f_{1}\left(x_{k}\right), f_{2}\left(x_{k}\right), f_{3}\left(x_{k}\right)\right)$ for $k=0,1, \ldots, N_{x}$.

3. For $n=0,1, \ldots, N_{t}$ :

i. apply $U_{k}^{n+1}=U_{k}^{n} \oplus \mu\left(U_{k+1}^{n} \ominus 2 U_{k}^{n} \oplus U_{k-1}^{n}\right) \ominus(-1) \Delta t F_{k}^{n}$ for all $k=1, \ldots, N_{x}-$ 1.

ii. set the boundary value $U_{0}^{n+1}=\left(g_{1}\left(t_{n+1}\right), g_{2}\left(t_{n+1}\right), g_{3}\left(t_{n+1}\right)\right)$.

iii. set the boundary value $U_{N_{x}}^{n+1}=\left(h_{1}\left(t_{n+1}\right), h_{2}\left(t_{n+1}\right), h_{3}\left(t_{n+1}\right)\right)$.

\section{Numerical Examples}

We will solve a few examples of the fuzzy finite difference method in this section to illustrate its efficiency and accuracy in solving the fuzzy heat equation. All calculations were performed on a PC running Mathematica software.

In the following example, the fuzzy finite difference method is explained in detail.

Example 51 (Numerical illustration) Consider the following initial-boundary non-homogeneous fuzzy heat equation

$$
\left\{\begin{array}{l}
u_{t_{i . g H}}=u_{x x_{i . g H}} \oplus(2 x, 3 x, 5 x), \\
u(x, 0)=(2 x, 3 x, 5 x), \\
u(0, t)=0, \quad u(2, t)=(4 t+4,6 t+6,10 t+10)
\end{array} \quad x \in[0,2], t \in[0,1] ;\right.
$$

This equation has exact fuzzy solution $u(x, t)=(2(t+1) x, 3(t+1) x, 5(t+1) x)$.

Considering Algorithm 41, step-by-step procedure to solve the given example is as follows

1. Suppose $N_{x}=2$ and $N_{t}=3$, then $\frac{\Delta t}{\Delta x^{2}}=\frac{1}{3}$ and

$$
\begin{aligned}
& t_{0}=0, \quad t_{1}=\frac{1}{3}, \quad t_{2}=\frac{2}{3}, \quad t_{3}=1, \\
& x_{0}=0, \quad x_{1}=1, \quad x_{2}=2 .
\end{aligned}
$$


2. For $k=0,1,2$

$$
U_{k}^{0}=\left(2\left(x_{k}\right), 3\left(x_{k}\right), 5\left(x_{k}\right)\right)
$$

Therefore

$$
U_{0}^{0}=(0,0,0), \quad U_{1}^{0}=(2,3,5), \quad U_{2}^{0}=(4,6,10) .
$$

3. For $n=0,1,2$

$$
\begin{aligned}
& U_{k}^{n+1}=U_{k}^{n} \oplus \mu\left(U_{k+1}^{n} \ominus 2 U_{k}^{n} \oplus U_{k-1}^{n}\right) \oplus \Delta t F_{k}^{n}, \quad k=1 \\
& U_{N_{x}}^{n+1}=\left(4 t_{n+1}+4,6 t_{n+1}+6,10 t_{n+1}+10\right), \\
& U_{0}^{n+1}=(0,0,0) .
\end{aligned}
$$

Hence

$$
\begin{aligned}
& -\mathrm{n}=0 \\
& U_{1}^{1}=U_{1}^{0} \oplus \mu\left(U_{2}^{0} \ominus 2 U_{1}^{0} \oplus U_{0}^{0}\right) \oplus \Delta t F_{1}^{0} \\
& =(2,3,5) \oplus \frac{1}{3}((4,6,10) \ominus 2(2,3,5) \oplus(0,0,0)) \oplus \frac{1}{3}\left(2\left(x_{1}\right), 3\left(x_{1}\right), 5\left(x_{1}\right)\right) \\
& =(2,3,5) \oplus \frac{1}{3}(2,3,5)=\left(\frac{8}{3}, 4, \frac{20}{3}\right) \text {. } \\
& U_{2}^{1}=\left(4 t_{1}+4,6 t_{1}+6,10 t_{1}+10\right)=\left(\frac{16}{3}, 8, \frac{40}{3}\right) \text {. } \\
& U_{0}^{1}=(0,0,0) \text {. } \\
& -n=1 \\
& \begin{array}{l}
U_{1}^{2}=U_{1}^{1} \oplus \mu\left(U_{2}^{1} \ominus 2 U_{1}^{1} \oplus U_{0}^{1}\right) \oplus \Delta t F_{1}^{1}=\left(\frac{10}{3}, 5, \frac{25}{3}\right) . \\
U_{2}^{2}=\left(4 t_{2}+4,6 t_{2}+6,10 t_{2}+10\right)=\left(\frac{20}{3}, 10, \frac{50}{3}\right) . \\
U_{0}^{2}=(0,0,0)
\end{array} \\
& -\mathrm{n}=2 \\
& U_{1}^{3}=U_{1}^{2} \oplus \mu\left(U_{2}^{2} \ominus 2 U_{1}^{2} \oplus U_{0}^{2}\right) \oplus \Delta t F_{1}^{2}=(4,6,10) . \\
& U_{2}^{3}=\left(4 t_{3}+4,6 t_{3}+6,10 t_{3}+10\right)=(8,12,20) \text {. } \\
& U_{0}^{3}=(0,0,0) \text {. }
\end{aligned}
$$

By placing the values $\left(x_{k}, t_{n}\right)$ in the exact solution, it is easy to verify $U_{k}^{n}=$ $u\left(x_{k}, t_{n}\right)$. Then the exact solution of the fuzzy heat equation (18) is obtained by this method. 
Example 52 Consider the following initial-boundary fuzzy heat equation

$$
\left\{\begin{array}{l}
u_{t_{i . g H}}=u_{x x_{i . g H}} \oplus\left(x e^{t}, 3.5 x e^{t}, 4.5 x e^{t}\right), x \in[0,1], t \in[0,1] \\
u(x, 0)=(x, 3.5 x, 4.5 x) \\
u(0, t)=(0,0,0) \\
u(2, t)=\left(e^{t}, 3.5 e^{t}, 4.5 e^{t}\right) .
\end{array}\right.
$$

Note that the exact solution of this equation is $u(x, t)=\left(x e^{t}, 3.5 x e^{t}, 4.5 x e^{t}\right)$. Let $N_{x}=2$ and $N_{t}=10$. The numerical results are shown in Table 1 . In addition, the approximate solution, $U_{1}^{n+1}$, and the exact solution, $u\left(x_{1}, t_{n+1}\right)$, are shown in Figures 1 when $x_{1}=\frac{1}{2}, n=0,1, \ldots, N_{t}$ and for all $\alpha \in[0,1]$. We observe that the fuzzy finite difference method is an accurate method for solving the given fuzzy heat equation.

\begin{tabular}{ccc}
\hline$x_{i}$ & $u\left(x_{k}, t_{n}\right)$ & $U_{k}^{n}$ \\
\hline$\left(\frac{1}{2}, 0.1\right)$, & $(0.5525,1.9340,2.4866)$ & $(0.55,1.925,2.475)$ \\
$\left(\frac{1}{2}, 0.2\right)$ & $(0.6107,2.1374,2.7481)$ & $(0.6103,2.1356,2.7429)$ \\
$\left(\frac{1}{2}, 0.3\right)$ & $(0.67492,2.3622,3.0371)$ & $(0.6710,2.3688,3.0399)$ \\
$\left(\frac{1}{2}, 0.4\right)$ & $(0.7459,2.6106,3.3566)$ & $(0.7416,2.6158,3.3574)$ \\
$\left(\frac{1}{2}, 0.5\right)$ & $(0.8243,2.8852,3.7096)$ & $(0.8196,2.8857,3.7884)$ \\
$\left(\frac{1}{2}, 0.6\right)$ & $(0.9110,3.1887,4.0997)$ & $(0.9158,3.1804,4.0863)$ \\
$\left(\frac{1}{2}, 0.7\right)$ & $(1.0068,3.5240,4.5309)$ & $(1.0051,3.5339,4.5309)$ \\
$\left(\frac{1}{2}, 0.8\right)$ & $(1.1127,3.8947,5.0074)$ & $(1.11641,3.8824,4.9788)$ \\
$\left(\frac{1}{2}, 0.9\right)$ & $(1.2298,4.3043,5.5341)$ & $(1.2227,4.3097,5.5024)$ \\
$\left(\frac{1}{2}, 1\right)$ & $(1.3591,4.7569,6.1161)$ & $(1.3513,4.7498,6.1811)$ \\
\hline
\end{tabular}

Table 1 Exact and approximate values by finite difference method for Example 52 

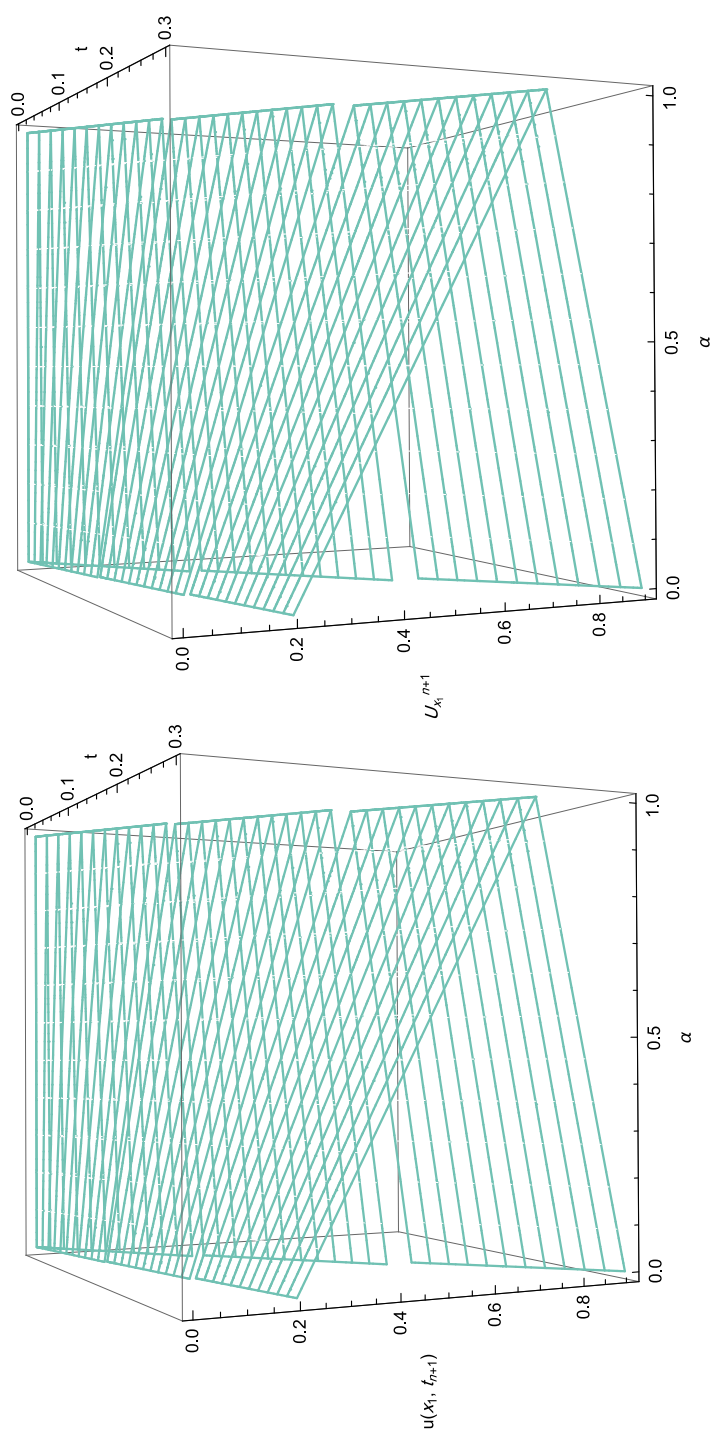

Fig. 1 Graphs of fuzzy-valued functions of $u\left(x_{1}, t_{n+1}\right)$ (Right) and $U_{1}^{n+1}$ (Left) of Example 52 .

Example 53 Consider the following fuzzy PDE

$$
u_{t_{i i . g H}}=u_{x x_{i i . g H}}, x \in[0,0.5], t \in[0,1]
$$

where $u(x, 0)=(\sin (\pi x), 3 \sin (\pi x), 5 \sin (\pi x)), u(0, t)=(0,0,0)$ and $u(0.5, t)=$ $\left(e^{-\pi^{2} t}, 3 e^{-\pi^{2} t}, 5 e^{-\pi^{2} t}\right)$ with exact solution $u(x, t)=\left(\sin (\pi x) e^{-\pi^{2} t}, 3 \sin (\pi x) e^{-\pi^{2} t}, 5 \sin (\pi x) e^{-\pi^{2} t}\right)$.

According to the procedure outlined in Algorithm 42, $\Delta t$ and $\Delta x$ should be consider large such that $\frac{\Delta t}{\Delta x^{2}} \leq \frac{1}{2}$. We consider $N_{x}=2$ and $N_{t}=50$. So we have 
many sub-intervals and it is not possible to show the approximate numbers, $U_{j}^{n}$, and only the approximate and exact solution are shown in Figures 2. In addition, Figures 3 represent the logarithm of the error for various $N_{t}$.
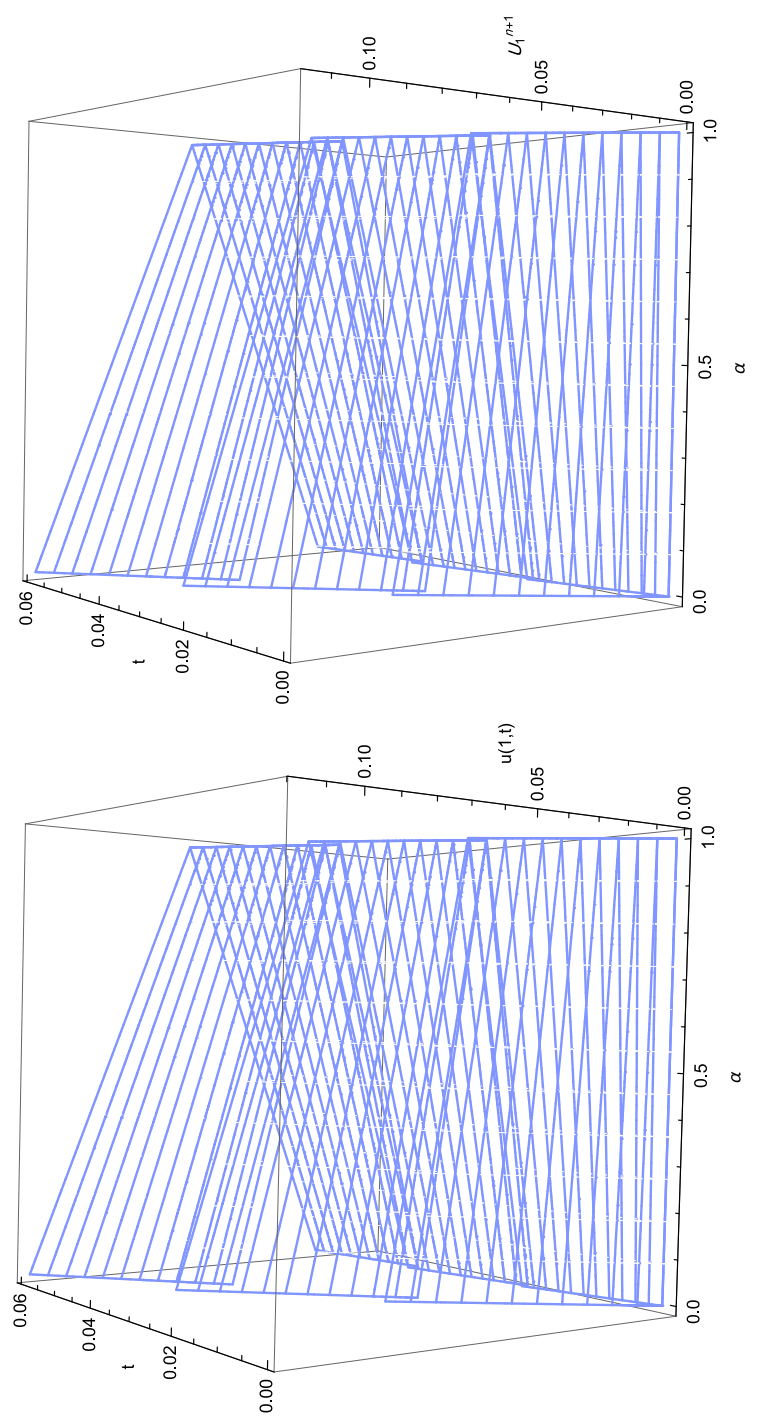

Fig. 2 Graphs of $u\left(x_{1}, t^{n+1}\right)$ (Right) and $U_{1}^{n+1}$ (Left) for Example 53 

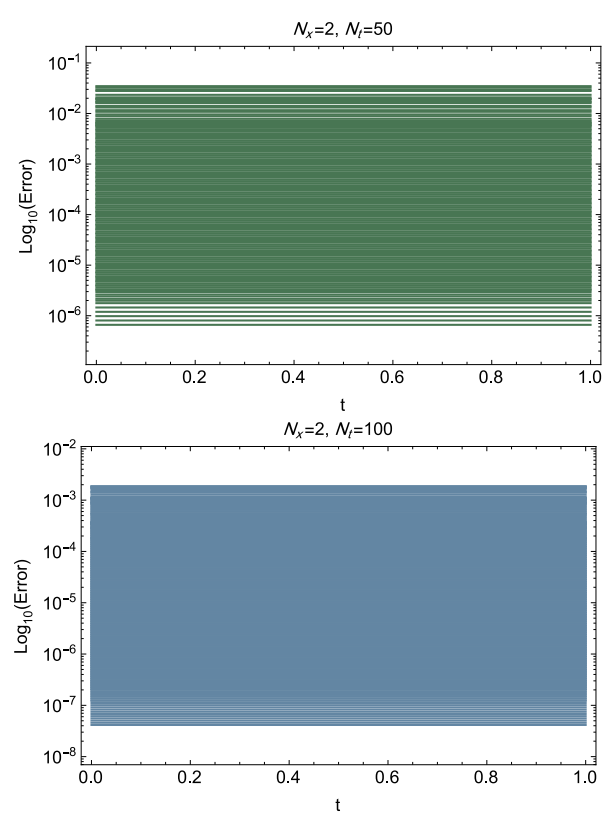

Fig. 3 Graph of the finite difference approximation error of Example 53.

\section{Conclusion}

We presented the new fuzzy finite difference method for approximating the fuzzy triangular solution of the fuzzy non-homogeneous heat equation with triangular initial-boundary conditions. To do this, the fuzzy Taylor expansion was extended according to the type of $g H$-differentiability, and the finite difference formulas for the first and second derivatives of a triangular fuzzy function $y(t)$ were obtained. Moreover, the convergence conditions for solving the fuzzy heat problem were also investigated. Several numerical examples were presented to demonstrate the performance of the methods. The final results demonstrated the efficiency and the ability of the new fuzzy finite difference method to produce triangular fuzzy numerical results which are more consistent with existing reality. Even though this paper deals with the fuzzy non-homogeneous heat equation, our method can be used to find the numerical solution for a wide variety of fuzzy partial differential equations. The fuzzy numerical solution of the fuzzy partial differential equations can be obtained without implicitly embedding them into crisp equations through our method.

Acknowledgements The authors would like to express deep gratitude to the editors and referees for their valuable suggestions which led us to a better presentation of this paper. 
Compliance with Ethical Standards

Conflict of interest

The authors declare that they have no conflict of interest.

\section{Ethical approval}

This article does not contain any studies with human participants or animals performed by any of the authors.

\section{References}

1. Allahviranloo, T.(2002) Difference methods for fuzzy partial differential equations.CMAM, 2,233-242.

2. Allahviranloo, T., Taheri,N.(2009) An Analytic Approximation to the Solution of Fuzzy Heat Equation by Adomian Decomposition Method, Int. J. Contemp. Math. Sciences, 4,105-114.

3. Allahviranloo,T., Gouyandeh, Z., Armand,A.(2015) A full fuzzy method for solving differential equation based on Taylor expansion, Journal of Intelligent and Fuzzy Systems 29, 1039-1055.

4. Allahviranloo, T., Afshar, K.M.(2010) Numerical methods for fuzzy linear partial differential equations under new definition for derivative. Iran. J. Fuzzy Syst. 7(3), 33-50.

5. Allahviranloo, T., Gouyandeh, Z., Armand, A., Hasanoglu, A.(2015) On fuzzy solutions for heat equation based on generalized hukuhara differentiability. Fuzzy Sets and Systems, 265,1-23,

6. Alikhani, R., Bahrami, F.(2019) Fuzzy partial differential equations under the cross product of fuzzy numbers, Information Sciences,494,80-99, doi: https://doi.org/10.1016/j.ins.2019.04.030.

7. Bede, B. , Gal, S.G. (2005) Generalizations of the differentiability of fuzzy-number-valued functions with applications to fuzzy differential equations, Fuzzy Set and Systems, 151, 581-599.

8. Bede, B., Gal, S.G. (2006) Remark on the new solutions of fuzzy differential equations, Chaos Solitons Fractals.

9. Bede, B. (2013) Mathematics of fuzzy sets and fuzzy logic, Springer, London.

10. Bertone, A. M. , Jafelice, R. M., de Barros,L. C., Bassanezi,R. C.(2013) On fuzzy solutions for partial differential equations, Fuzzy Sets and Systems, 219, 68-80.

11. Buckley, J.J., Feuring, T. (1999) Introduction to fuzzy partial differential equations, Fuzzy Sets and Systems, 105, 241-248.

12. Chalco-Cano, Y., Roman-Flores, H., Jimenez-Gamero, M.D. (2011) Generalized derivative and $\pi$-derivative for set-valued functions, Information Sciences, 181, 2177-2188.

13. Chalco-Cano, Y., Costab, T.M., Román-Floresc, H., Rufián-Lizana, A. (2020) New properties of the switching points for the generalized Hukuhara differentiability and some results on calculus, Fuzzy Sets and Systems,https://doi.org/10.1016/j.fss.2020.06.016.

14. Chang, S., Zadeh, L. (1972) On fuzzy mapping and control, IEEE Trans Syst Cybern, $2,30-34$.

15. Diamond, P. (2002)Brief note on the variation of constants formula for fuzzy differential equations, Fuzzy Sets Syst, 129, 65-71.

16. Hukuhara, M. (1967) Integration des applications mesurables dont la valeur est un compact convex, Funkcial Ekvac, 10, 205-229.

17. Gouyandeh, Z., Allahviranloo, T., Abbasbandy, S., Armand, A.(2017) A fuzzy solution of heat equation under generalized Hukuhara differentiability by fuzzy Fourier transform, Fuzzy Sets and System,309,81-97. 
18. Kaleva, O. (1987) Fuzzy differential equations, Fuzzy Sets and Systems, 24, 301-317.

19. Kaufmann, A., Gupta, M.M.(1985) Introduction Fuzzy Arithmetic, Van Nostrand Reinhold, New York

20. Lakshmikantham,V., Bhaskar, T., Devi, J. (2006) Theory of Set Differential Equations in Metric Spaces, Cambridge Scientific Publishers.

21. Mahmoud, M.M., Iman, J.(2011) Finite volume methods for fuzzy parabolic equations. J. Math. Comput. Sci. 2(3), 546-558.

22. Moghaddam, R. G., Allahviranloo, T. (2018) On the fuzzy Poisson equation, Fuzzy Sets Syst., 347, 105-128

23. Puri, M.L.,Ralescu, D.A. (1986) Differentials of fuzzy functions, J Math Anal Appl, 114 409-422.

24. Pirzada, U.M., Vakaskar, D.C.(2015) Solution of fuzzy heat equations using adomian decomposition method. Int. J. Adv. Appl. Math. Mech. 3(1), 87-91.

25. Stefanini, L., Bede, B. (2009) Generalized Hukuhara differentiability of interval-valued functions and interval differential equations, Nonlinear Analysis, 71, 1311-1328. 